\title{
On the Unilateral Contact Between Membranes Part 1: Finite Element Discretization and Mixed Reformulation
}

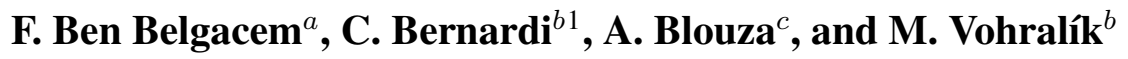 \\ ${ }^{a}$ L.M.A.C. (E.A. 2222), Département de Génie Informatique, \\ Université de Technologie de Compiègne, Centre de Recherches de Royallieu, \\ B.P. 20529, 60205 Compiègne Cedex, France. \\ ${ }^{b}$ Laboratoire Jacques-Louis Lions, C.N.R.S. \& Université Pierre et Marie Curie, \\ B.C. 187, 4 place Jussieu, 75252 Paris Cedex 05, France. \\ ${ }^{c}$ Laboratoire de Mathématiques Raphaël Salem (U.M.R. 6085 C.N.R.S.), Université de Rouen, \\ avenue de l'Université, B.P. 12, 76801 Saint-Étienne-du-Rouvray, France.
}

\begin{abstract}
The contact between two membranes can be described by a system of variational inequalities, where the unknowns are the displacements of the membranes and the action of a membrane on the other one. We first perform the analysis of this system. We then propose a discretization, where the displacements are approximated by standard finite elements and the action by a local postprocessing. Such a discretization admits an equivalent mixed reformulation. We prove the well-posedness of the discrete problem and establish optimal a priori error estimates.
\end{abstract}

Key words: unilateral contact, elastic membranes, variational inequalities.

AMS subject classification: 65N30, 73K10, $73 \mathrm{~T} 05$.

\footnotetext{
${ }^{1}$ Corresponding author. E-mail: bernardi@ann.jussieu.fr
} 


\section{Introduction}

We are interested in the discretization of the following system, set in a bounded open set $\omega$ in $\mathbb{R}^{2}$ with a Lipschitz-continuous boundary:

$$
\begin{cases}-\mu_{1} \Delta u_{1}-\lambda=f_{1} & \text { in } \omega \\ -\mu_{2} \Delta u_{2}+\lambda=f_{2} & \text { in } \omega, \\ u_{1}-u_{2} \geq 0, \quad \lambda \geq 0, \quad\left(u_{1}-u_{2}\right) \lambda=0 & \text { in } \omega, \\ u_{1}=g & \text { on } \partial \omega \\ u_{2}=0 & \text { on } \partial \omega .\end{cases}
$$

Indeed, such a system is a model for the contact between two membranes and can easily be derived from the fundamental laws of elasticity (more details are given in [2, §2]). In this model, the unknowns are the displacements $u_{1}$ and $u_{2}$ of the two membranes and the Lagrange multiplier $\lambda$ which represents the action of the second membrane on the first one (so that $-\lambda$ is the reaction). The coefficients $\mu_{1}$ and $\mu_{2}$ are positive constants corresponding to the tension of the membranes. The data are the external forces $f_{1}$ and $f_{2}$ and also the boundary datum $g$ : Indeed the boundary conditions in system (1.1) mean that the first membrane is fixed on $\partial \omega$ at the height $g$, where $g$ is a nonnegative function, and the second one is fixed at zero. This kind of system appears in a large number of problems in elasticity, such as the obstacle or Signorini problems, see [6, Chap. 5] and [7] among others. Finite element discretizations of variational inequalities have also been analyzed in a number of works, see [5], [9], [10], and the references therein.

The analysis of problem (1.1) is performed in [2] in the case of homogeneous boundary data $g=0$, where the action $\lambda$ is implicitly linked to a displacement. Here, we consider the case where $g \neq 0$. Thus, we are led to write a new variational formulation for problem (1.1), where $\lambda$ is explicitly taken into account, which requires more regularity to give sense to the complementarity equation $\left(u_{1}-u_{2}\right) \lambda=0$. As standard for mixed problems, the displacements $u_{1}$ and $u_{2}$ are the solution of a reduced variational inequality. We prove the well-posedness successively of the reduced problem, next of the full problem.

The discretization of problem (1.1) is made in two steps. In a first step, we propose a finite element discretization of the reduced problem, prove that the discrete problem is well-posed, and establish optimal a priori estimates under minimal regularity assumptions. The discretization of the full problem relies on the reduced discrete problem but is more complex. We propose a discrete problem that requires the introduction of a dual mesh and can be interpreted as a finite volume scheme. The corresponding discrete problem is well-posed, and optimal a priori error estimates are also derived.

The a posteriori analysis of our discrete problem is under consideration, together with some numerical experiments.

An outline of the paper is as follows. 
- Section 2 is devoted to the analysis of system (1.1).

- Sections 3 and 4 deal with the reduced and full discrete problems, respectively. In both cases, we check their well-posedness and prove a priori error estimates.

\section{Analysis of the continuous problem}

We first write a variational formulation of system (1.1). In order to do this, we consider the full scales of Sobolev spaces $H^{s}(\omega)$ and $H^{s}(\partial \omega), s \geq 0$, equipped with the usual norms (and seminorms when $s$ is a positive integer). We also need the space $H_{0}^{1}(\omega)$ of functions in $H^{1}(\omega)$ which vanish on $\partial \omega$ and, for any function $g$ in $H^{\frac{1}{2}}(\partial \omega)$, the space

$$
H_{g}^{1}(\omega)=\left\{v \in H^{1}(\omega) ; v=g \text { on } \partial \omega\right\} .
$$

Next, we introduce the convex subset

$$
\Lambda=\left\{\chi \in L^{2}(\omega) ; \chi \geq 0 \text { a.e. in } \omega\right\}
$$

and, in order to take into account the nonnegativity of the boundary condition $g$, the cones defined for each $s \geq 0$ by

$$
H_{+}^{s}(\partial \omega)=\left\{k \in H^{s}(\partial \omega) ; k \geq 0 \text { a.e. in } \partial \omega\right\} .
$$

So we consider the following variational problem, for any data $\left(f_{1}, f_{2}\right)$ in $H^{-1}(\omega) \times H^{-1}(\omega)$ and $g$ in $H_{+}^{\frac{1}{2}}(\partial \omega)$ :

Find $\left(u_{1}, u_{2}, \lambda\right)$ in $H_{g}^{1}(\omega) \times H_{0}^{1}(\omega) \times \Lambda$ such that

$$
\begin{aligned}
& \begin{array}{c}
\forall\left(v_{1}, v_{2}\right) \in H_{0}^{1}(\omega) \times H_{0}^{1}(\omega), \quad \sum_{i=1}^{2} \mu_{i} \int_{\omega}\left(\operatorname{grad} u_{i}\right)(\boldsymbol{x}) \cdot\left(\operatorname{grad} v_{i}\right)(\boldsymbol{x}) \mathrm{d} \boldsymbol{x} \\
-\int_{\omega} \lambda(\boldsymbol{x})\left(v_{1}-v_{2}\right)(\boldsymbol{x}) \mathrm{d} \boldsymbol{x}=\sum_{i=1}^{2}\left\langle f_{i}, v_{i}\right\rangle, \\
\forall \chi \in \Lambda, \quad \int_{\omega}(\chi-\lambda)(\boldsymbol{x})\left(u_{1}-u_{2}\right)(\boldsymbol{x}) \mathrm{d} \boldsymbol{x} \geq 0 .
\end{array}
\end{aligned}
$$

We must now check the equivalence of this problem with system (1.1).

Proposition 1. Problems (1.1) and (2.4) are equivalent, in the sense that any triple $\left(u_{1}, u_{2}, \lambda\right)$ in $H^{1}(\omega) \times H^{1}(\omega) \times L^{2}(\omega)$ is a solution of (1.1) if and only if it is a solution of (2.4).

Proof: Since the fourth and fifth lines in (1.1) are obviously equivalent to the fact that $u_{1}$ and $u_{2}$ belong to $H_{g}^{1}(\omega)$ and $H_{0}^{1}(\omega)$, respectively, we now verify the equivalence of the other lines.

1) Let $\mathcal{D}(\omega)$ be the space of infinitely differentiable functions with a compact support in $\omega$. Multiplying the first line of (1.1) by a function $v_{1}$ in $\mathcal{D}(\omega)$ and the second line by a function $v_{2}$ in $\mathcal{D}(\omega)$, summing these two equations, and integrating by parts yield that the first equation in (2.4) is satisfied for all pairs $\left(v_{1}, v_{2}\right)$ in $\mathcal{D}(\omega)^{2}$. Thus, it follows from the density of $\mathcal{D}(\omega)$ in $H_{0}^{1}(\omega)$ that this 
line is satisfied for all $\left(v_{1}, v_{2}\right)$ in $H_{0}^{1}(\omega)^{2}$. Conversely, by letting $v_{1}$ run through $\mathcal{D}(\omega)$ and taking $v_{2}$ equal to zero, next by taking $v_{1}$ equal to zero and letting $v_{2}$ run through $\mathcal{D}(\omega)$, we observe that the first line of (2.4) implies the first two lines of (1.1) in the sense of distributions.

2) Let $\left(u_{1}, u_{2}, \lambda\right)$ satisfy the third line of (1.1). Thus, $\lambda$ belongs to $\Lambda$ and it follows from the definition of $\Lambda$ that, for all $\chi$ in $\Lambda$,

$$
\int_{\omega}(\chi-\lambda)(\boldsymbol{x})\left(u_{1}-u_{2}\right)(\boldsymbol{x}) \mathrm{d} \boldsymbol{x}=\int_{\omega} \chi(\boldsymbol{x})\left(u_{1}-u_{2}\right)(\boldsymbol{x}) \mathrm{d} \boldsymbol{x} \geq 0 .
$$

Conversely, if $\lambda$ belongs to $\Lambda$ and $\left(u_{1}, u_{2}, \lambda\right)$ satisfies the second line of (2.4), taking $\chi$ equal to the sum of $\lambda$ and of the characteristic function $\chi_{\mathcal{O}}$ of any measurable subset $\mathcal{O}$ of $\omega$ (this $\chi_{\mathcal{O}}$ obviously belongs to $\Lambda$ ) yields that

$$
\int_{\mathcal{O}}\left(u_{1}-u_{2}\right)(\boldsymbol{x}) \mathrm{d} \boldsymbol{x} \geq 0
$$

whence the nonnegativity of $u_{1}-u_{2}$. Finally, taking $\chi$ equal to zero yields that

$$
\int_{\omega} \lambda(\boldsymbol{x})\left(u_{1}-u_{2}\right)(\boldsymbol{x}) \mathrm{d} \boldsymbol{x} \leq 0
$$

and combining this with the previous properties gives the equality $\left(u_{1}-u_{2}\right) \lambda=0$.

Setting $\boldsymbol{u}=\left(u_{1}, u_{2}\right)$ and $\boldsymbol{v}=\left(v_{1}, v_{2}\right)$, we consider the bilinear form defined by

$$
a(\boldsymbol{u}, \boldsymbol{v})=\sum_{i=1}^{2} \mu_{i} \int_{\omega}\left(\operatorname{grad} u_{i}\right)(\boldsymbol{x}) \cdot\left(\operatorname{grad} v_{i}\right)(\boldsymbol{x}) \mathrm{d} \boldsymbol{x} .
$$

Its continuity on $H^{1}(\omega)^{2} \times H^{1}(\omega)^{2}$ is obvious and its ellipticity on $H_{0}^{1}(\omega)^{2}$ follows from the Poincaré-Friedrichs inequality: There exists a constant $\alpha>0$ only depending on $\omega$ and on the $\mu_{i}$ such that

$$
\forall \boldsymbol{v} \in H_{0}^{1}(\omega)^{2}, \quad a(\boldsymbol{v}, \boldsymbol{v}) \geq \alpha\|\boldsymbol{v}\|_{H^{1}(\omega)^{2}}^{2} .
$$

With the same notation, we also introduce the bilinear form

$$
b(\boldsymbol{v}, \chi)=-\int_{\omega} \chi(\boldsymbol{x})\left(v_{1}-v_{2}\right)(\boldsymbol{x}) \mathrm{d} \boldsymbol{x},
$$

which is continuous on $H^{1}(\omega)^{2} \times L^{2}(\omega)$.

Problem (2.4) fits the abstract framework introduced in [13]; however the inf-sup condition on the form $b(\cdot, \cdot)$ fails. So we have rather study it by hand. For this, we introduce the new convex set

$$
\mathcal{K}_{g}=\left\{\left(v_{1}, v_{2}\right) \in H_{g}^{1}(\omega) \times H_{0}^{1}(\omega) ; v_{1}-v_{2} \geq 0 \text { a.e. in } \omega\right\} .
$$

Since the function $g$ is nonnegative, this last set is not empty. We then consider the reduced problem 
Find $\left(u_{1}, u_{2}\right)$ in $\mathcal{K}_{g}$ such that

$$
\begin{aligned}
\forall\left(v_{1}, v_{2}\right) \in \mathcal{K}_{g}, \quad \sum_{i=1}^{2} \mu_{i} \int_{\omega}\left(\operatorname{grad} u_{i}\right)(\boldsymbol{x}) \cdot\left(\operatorname{grad}\left(v_{i}-u_{i}\right)\right)(\boldsymbol{x}) \mathrm{d} \boldsymbol{x} & \\
& \geq \sum_{i=1}^{2}\left\langle f_{i}, v_{i}-u_{i}\right\rangle .
\end{aligned}
$$

The reason for this is stated in the next lemma.

Lemma 2. For any solution $\left(u_{1}, u_{2}, \lambda\right)$ of problem (2.4), the pair $\left(u_{1}, u_{2}\right)$ is a solution of problem (2.9).

Proof: Let $\left(u_{1}, u_{2}, \lambda\right)$ be a solution of problem (2.4). Owing to Proposition 1, it satisfies the third line of (1.1), so that $\left(u_{1}, u_{2}\right)$ belongs to $\mathcal{K}_{g}$. On the other hand, since $\Lambda$ is a closed convex cone, we have

$$
\int_{\omega} \lambda(\boldsymbol{x})\left(u_{1}-u_{2}\right)(\boldsymbol{x}) \mathrm{d} \boldsymbol{x}=0 .
$$

Finally, it can be noted that, for any $\left(v_{1}, v_{2}\right)$ in $\mathcal{K}_{g}$, the pair $\left(v_{1}-u_{1}, v_{2}-u_{2}\right)$ belongs to $H_{0}^{1}(\omega) \times$ $H_{0}^{1}(\omega)$. Thus, replacing each $v_{i}$ by $v_{i}-u_{i}$ in problem (2.4) and using the previous inequalities lead to $(2.9)$.

Owing to the ellipticity property (2.6), the existence and uniqueness of a solution for problem (2.9) is now a direct consequence of the Lions-Stampacchia theorem [11].

Proposition 3. For any data $\left(f_{1}, f_{2}\right)$ in $H^{-1}(\omega) \times H^{-1}(\omega)$ and $g$ in $H_{+}^{\frac{1}{2}}(\partial \omega)$, problem (2.9) has a unique solution $\left(u_{1}, u_{2}\right)$ in $\mathcal{K}_{g}$.

We now prove a further regularity of the solution $\left(u_{1}, u_{2}\right)$. The arguments are the same as in [4] but simpler, so that we prefer to give a direct proof. For any function $v$ in $H^{1}(\omega)$, we denote by $v_{-}$the function $\min \{v, 0\}$.

Proposition 4. For any data $\left(f_{1}, f_{2}\right)$ in $L^{2}(\omega) \times L^{2}(\omega)$, the solution $\left(u_{1}, u_{2}\right)$ of problem $(2.9)$ is such that $\left(-\Delta u_{1},-\Delta u_{2}\right)$ belongs to $L^{2}(\omega) \times L^{2}(\omega)$. Moreover, the following property holds

$$
\mu_{1}\left\|\Delta u_{1}\right\|_{L^{2}(\omega)}+\mu_{2}\left\|\Delta u_{2}\right\|_{L^{2}(\omega)} \leq c\left(\left\|f_{1}\right\|_{L^{2}(\omega)}+\left\|f_{2}\right\|_{L^{2}(\omega)}\right) .
$$

Proof: For any $\varepsilon>0$, the problem: Find $u_{\varepsilon 1}$ in $H_{g}^{1}(\omega)$ and $u_{\varepsilon 2}$ in $H_{0}^{1}(\omega)$ such that

$$
u_{\varepsilon i}-\varepsilon \Delta u_{\varepsilon i}=u_{i}, \quad i=1,2,
$$

has a unique solution. Moreover, it admits the following variational formulation

$$
\begin{aligned}
\forall v \in H_{0}^{1}(\omega), \quad \int_{\omega} u_{\varepsilon i}(\boldsymbol{x}) v(\boldsymbol{x}) \mathrm{d} \boldsymbol{x}+\varepsilon \int_{\omega}\left(\operatorname{grad} u_{\varepsilon i}\right)(\boldsymbol{x})(\operatorname{grad} v)(\boldsymbol{x}) \mathrm{d} \boldsymbol{x} \\
=\int_{\omega} u_{i}(\boldsymbol{x}) v(\boldsymbol{x}) \mathrm{d} \boldsymbol{x}, \quad i=1,2 .
\end{aligned}
$$


1) Choosing $v$ equal to $\left(u_{\varepsilon 1}-u_{\varepsilon 2}\right)_{-}$in these equations (since $g$ is nonnegative, this function belongs to $\left.H_{0}^{1}(\omega)\right)$ and subtracting the equation for $i=2$ from the equation for $i=1$, we derive

$$
\left\|\left(u_{\varepsilon 1}-u_{\varepsilon 2}\right)_{-}\right\|_{L^{2}(\omega)}^{2}+\varepsilon\left|\left(u_{\varepsilon 1}-u_{\varepsilon 2}\right)_{-}\right|_{H^{1}(\omega)}^{2}=\int_{\omega}\left(u_{1}-u_{2}\right)(\boldsymbol{x})\left(u_{\varepsilon 1}-u_{\varepsilon 2}\right)_{-}(\boldsymbol{x}) \mathrm{d} \boldsymbol{x} .
$$

Since the right-hand side of this equation is nonpositive, we derive that $\left(u_{\varepsilon 1}-u_{\varepsilon 2}\right)_{-}$is zero, so that the pair $\left(u_{\varepsilon 1}, u_{\varepsilon 2}\right)$ belongs to $\mathcal{K}_{g}$.

2) When taking $v_{i}$ equal to $u_{\varepsilon i}$ in problem (2.9), we observe that

$$
\sum_{i=1}^{2} \mu_{i} \int_{\omega}\left(\operatorname{grad} u_{i}\right)(\boldsymbol{x}) \cdot\left(\operatorname{grad}\left(u_{i}-u_{\varepsilon i}\right)\right)(\boldsymbol{x}) \mathrm{d} \boldsymbol{x} \leq \sum_{i=1}^{2}\left\langle f_{i}, u_{i}-u_{\varepsilon i}\right\rangle
$$

or equivalently

$$
\sum_{i=1}^{2} \mu_{i}\left(\left|u_{i}-u_{\varepsilon i}\right|_{H^{1}(\omega)}^{2}+\int_{\omega}\left(\operatorname{grad} u_{\varepsilon i}\right)(\boldsymbol{x}) \cdot\left(\operatorname{grad}\left(u_{i}-u_{\varepsilon i}\right)\right)(\boldsymbol{x}) \mathrm{d} \boldsymbol{x}\right) \leq \sum_{i=1}^{2}\left\langle f_{i}, u_{i}-u_{\varepsilon i}\right\rangle .
$$

It thus follows from the variational formulation (2.12) that

$$
\varepsilon^{-1} \sum_{i=1}^{2} \mu_{i}\left\|u_{i}-u_{\varepsilon i}\right\|_{L^{2}(\omega)} \leq c \sum_{i=1}^{2}\left\|f_{i}\right\|_{L^{2}(\omega)} .
$$

So, the $u_{\varepsilon i}$ tend to $u_{i}$ strongly in $L^{2}(\omega)$ when $\varepsilon$ tends to zero.

3) On the other hand, it follows from the previous estimate that

$$
\sum_{i=1}^{2} \mu_{i}\left\|\Delta u_{\varepsilon i}\right\|_{L^{2}(\omega)} \leq c \sum_{i=1}^{2}\left\|f_{i}\right\|_{L^{2}(\omega)}
$$

Therefore, there exists a subsequence of the $u_{\varepsilon i}$ such that $\Delta u_{\varepsilon i}$ converges weakly in $L^{2}(\omega)$. Since its limit is necessarily $\Delta u_{i}$, each $\Delta u_{i}$ belongs to $L^{2}(\omega)$. Moreover, by combining the previous inequality with the convexity of the norm $\|\cdot\|_{L^{2}(\omega)}$, we obtain (2.10).

Thanks to Proposition 4, we are in a position to prove the main result of this section.

Theorem 5. For any data $\left(f_{1}, f_{2}\right)$ in $L^{2}(\omega) \times L^{2}(\omega)$ and $g$ in $H_{+}^{\frac{1}{2}}(\partial \omega)$, problem (2.4) has a unique solution $\left(u_{1}, u_{2}, \lambda\right)$ in $H_{g}^{1}(\omega) \times H_{0}^{1}(\omega) \times \Lambda$.

Proof: Let $\left(u_{1}, u_{2}\right)$ be the solution of problem (2.9). We set:

$$
\lambda_{1}=-\mu_{1} \Delta u_{1}-f_{1}, \quad \lambda_{2}=\mu_{2} \Delta u_{2}+f_{2} .
$$

1) It follows from Proposition 4 that $\lambda_{1}$ and $\lambda_{2}$ belong to $L^{2}(\omega)$. The variational form of the previous system reads, for all $v_{1}$ and $v_{2}$ in $H_{0}^{1}(\omega)$,

$$
\begin{aligned}
& \int_{\omega} \lambda_{1}(\boldsymbol{x}) v_{1}(\boldsymbol{x}) \mathrm{d} \boldsymbol{x}=\mu_{1} \int_{\omega}\left(\operatorname{grad} u_{1}\right)(\boldsymbol{x}) \cdot\left(\operatorname{grad} v_{1}\right)(\boldsymbol{x}) \mathrm{d} \boldsymbol{x}-\int_{\omega} f_{1}(\boldsymbol{x}) v_{1}(\boldsymbol{x}) \mathrm{d} \boldsymbol{x}, \\
& \int_{\omega} \lambda_{2}(\boldsymbol{x}) v_{2}(\boldsymbol{x}) \mathrm{d} \boldsymbol{x}=-\mu_{2} \int_{\omega}\left(\operatorname{grad} u_{2}\right)(\boldsymbol{x}) \cdot\left(\operatorname{grad} v_{2}\right)(\boldsymbol{x}) \mathrm{d} \boldsymbol{x}+\int_{\omega} f_{2}(\boldsymbol{x}) v_{2}(\boldsymbol{x}) \mathrm{d} \boldsymbol{x} .
\end{aligned}
$$


Subtracting the second equation from the first one and replacing each $v_{i}$ by $v_{i}-u_{i}$ for a pair $\left(v_{1}, v_{2}\right)$ in $\mathcal{K}_{g}$ lead to

$$
\begin{aligned}
& \int_{\omega} \lambda_{1}(\boldsymbol{x})\left(v_{1}-u_{1}\right)(\boldsymbol{x}) \mathrm{d} \boldsymbol{x}-\int_{\omega} \lambda_{2}(\boldsymbol{x})\left(v_{2}-u_{2}\right)(\boldsymbol{x}) \mathrm{d} \boldsymbol{x} \\
& =\sum_{i=1}^{2} \mu_{i} \int_{\omega}\left(\operatorname{grad} u_{i}\right)(\boldsymbol{x}) \cdot\left(\operatorname{grad}\left(v_{i}-u_{i}\right)\right)(\boldsymbol{x}) \mathrm{d} \boldsymbol{x}-\sum_{i=1}^{2} \int_{\omega} f_{i}(\boldsymbol{x})\left(v_{i}-u_{i}\right)(\boldsymbol{x}) \mathrm{d} \boldsymbol{x} .
\end{aligned}
$$

It follows from problem (2.9) that

$$
\int_{\omega} \lambda_{1}(\boldsymbol{x})\left(v_{1}-u_{1}\right)(\boldsymbol{x}) \mathrm{d} \boldsymbol{x}-\int_{\omega} \lambda_{2}(\boldsymbol{x})\left(v_{2}-u_{2}\right)(\boldsymbol{x}) \mathrm{d} \boldsymbol{x} \geq 0 .
$$

2) Let $\varphi$ be any function in $H_{0}^{1}(\omega)$. Then, the pairs $\left(v_{1}=u_{1} \pm \varphi, v_{2}=u_{2} \pm \varphi\right)$ belong to $\mathcal{K}_{g}$. Making this choice in (2.16) gives

$$
\int_{\omega}\left(\lambda_{1}-\lambda_{2}\right)(\boldsymbol{x}) \varphi(\boldsymbol{x}) \mathrm{d} \boldsymbol{x}=0
$$

It follows from the density of $\mathcal{D}(\omega)$ in $L^{2}(\omega)$ that there exists a sequence $\left(\varphi_{n}\right)_{n}$ in $H_{0}^{1}(\omega)$ which converges to $\lambda_{1}-\lambda_{2}$ in $L^{2}(\omega)$. Replacing $\varphi$ by $\varphi_{n}$ in the equation above and passing to the limit yield that $\left\|\lambda_{1}-\lambda_{2}\right\|_{L^{2}(\omega)}^{2}$ is zero. So, the functions $\lambda_{1}$ and $\lambda_{2}$ coincide with a unique function $\lambda$. Thus, the triple $\left(u_{1}, u_{2}, \lambda\right)$ satisfies the first line of problem (2.4).

3) We now consider a nonnegative function $\varphi$ in $H_{0}^{1}(\omega)$. Using once more (2.16) with $\left(v_{1}=\right.$ $\left.u_{1}+\varphi, v_{2}=u_{2}\right)$ implies

$$
\int_{\omega} \lambda(\boldsymbol{x}) \varphi(\boldsymbol{x}) \mathrm{d} \boldsymbol{x} \geq 0
$$

For any closed subset $\mathcal{O}$ of $\omega$ with positive measure, there exists a sequence $\left(\varphi_{n}\right)_{n}$ of nonnegative functions in $H_{0}^{1}(\omega)$ which converges to the characteristic function of $\mathcal{O}$ in $L^{2}(\omega)$. Taking $\varphi$ equal to $\varphi_{n}$ in the previous inequality and passing to the limit yield

$$
\int_{\mathcal{O}} \lambda(\boldsymbol{x}) \mathrm{d} \boldsymbol{x} \geq 0
$$

Thus, $\lambda$ is nonnegative and belongs to $\Lambda$.

4) Let $\left(\varphi_{n}\right)_{n}$ be a sequence of smooth functions with values in $[0,1]$, equal to 1 in a neighbourhood of $\partial \omega$ and such that the measure of the support of $\varphi_{n}$ is $\leq \frac{1}{n}$. By taking $\left(v_{1}=\varphi_{n} u_{1}, v_{2}=\varphi_{n} u_{2}\right)$, we derive from (2.16) that

$$
\int_{\omega} \lambda(\boldsymbol{x})\left(\varphi_{n}-1\right)(\boldsymbol{x})\left(u_{1}-u_{2}\right)(\boldsymbol{x}) \mathrm{d} \boldsymbol{x} \geq 0,
$$

whence, by letting $n$ tend to $+\infty$,

$$
-\int_{\omega} \lambda(\boldsymbol{x})\left(u_{1}-u_{2}\right)(\boldsymbol{x}) \mathrm{d} \boldsymbol{x} \geq 0 .
$$


On the other hand it follows from the definitions of $\Lambda$ and $\mathcal{K}_{g}$ that, for all $\chi$ in $\Lambda$,

$$
\int_{\omega} \chi(\boldsymbol{x})\left(u_{1}-u_{2}\right)(\boldsymbol{x}) \mathrm{d} \boldsymbol{x} \geq 0 .
$$

Summing the last two inequalities gives the second line of (2.4). As a consequence of all this, the triple $\left(u_{1}, u_{2}, \lambda\right)$ is a solution of (2.4).

5) Let $\left(u_{1}, u_{2}, \lambda\right)$ and $\left(\tilde{u}_{1}, \tilde{u}_{2}, \tilde{\lambda}\right)$ be two solutions of problem (2.4). Thus, owing to Lemma 2 , $\left(u_{1}, u_{2}\right)$ and $\left(\tilde{u}_{1}, \tilde{u}_{2}\right)$ are solutions of problem (2.9) and it follows from Proposition 3 that they coincide. Then, we deduce from problem (2.4) the equation

$$
\forall v \in H_{0}^{1}(\omega), \quad \int_{\Omega}(\lambda-\tilde{\lambda})(\boldsymbol{x}) v(\boldsymbol{x}) \mathrm{d} \boldsymbol{x}=0 .
$$

Using the density of $H_{0}^{1}(\omega)$ in $L^{2}(\omega)$ yields that $\lambda$ is equal to $\tilde{\lambda}$, whence the uniqueness of the solution of problem (2.4).

We also prove a stability estimate for this solution.

Corollary 6. For any data $\left(f_{1}, f_{2}\right)$ in $L^{2}(\omega) \times L^{2}(\omega)$ and $g$ in $H_{+}^{\frac{1}{2}}(\partial \omega)$, the solution $\left(u_{1}, u_{2}, \lambda\right)$ of problem (2.4) satisfies

$$
\left\|u_{1}\right\|_{H^{1}(\omega)}+\left\|u_{2}\right\|_{H^{1}(\omega)}+\|\lambda\|_{L^{2}(\omega)} \leq c\left(\left\|f_{1}\right\|_{L^{2}(\omega)}+\left\|f_{2}\right\|_{L^{2}(\omega)}+\|g\|_{H^{\frac{1}{2}}(\partial \omega)}\right) .
$$

Proof: We proceed in two steps.

1) Let $\bar{g}$ denote the harmonic lifting of the function $g$ : The function $\bar{g}$ is the solution in $H^{1}(\omega)$ of the problem

$$
\Delta \bar{g}=0 \quad \text { in } \omega, \quad \bar{g}=g \quad \text { on } \partial \omega
$$

and satisfies

$$
\|\bar{g}\|_{H^{1}(\omega)} \leq c\|g\|_{H^{\frac{1}{2}(\partial \omega)}} .
$$

Moreover, thanks to the maximum principle, $\bar{g}$ is nonnegative on $\omega$. Next, we take $v_{1}=u_{1}-\bar{g}$ and $v_{2}=u_{2}$ in the first line of problem (2.4). This yields

$$
\begin{array}{r}
\sum_{i=1}^{2} \mu_{i}\left|u_{i}\right|_{H^{1}(\omega)}^{2} \leq \sum_{i=1}^{2}\left\|f_{i}\right\|_{L^{2}(\omega)}\left\|u_{i}\right\|_{L^{2}(\omega)}+\left\|f_{1}\right\|_{L^{2}(\omega)}\|\bar{g}\|_{L^{2}(\omega)}+\mu_{1}\left|u_{1}\right|_{H^{1}(\omega)}|\bar{g}|_{H^{1}(\omega)} \\
+\int_{\omega} \lambda(\boldsymbol{x})\left(u_{1}-u_{2}\right)(\boldsymbol{x}) \mathrm{d} \boldsymbol{x}-\int_{\omega} \lambda(\boldsymbol{x}) \bar{g}(\boldsymbol{x}) \mathrm{d} \boldsymbol{x} .
\end{array}
$$

It follows from the second line of (2.4) with $\chi=0$, together with the definition of $\Lambda$ and the nonnegativity of $\bar{g}$, that the last two integrals in this inequality are nonpositive. On the other hand, we use the Poincaré-Friedrichs inequalities

$$
\begin{aligned}
& \left\|u_{1}\right\|_{L^{2}(\omega)} \leq\left\|u_{1}-\bar{g}\right\|_{L^{2}(\omega)}+\|\bar{g}\|_{L^{2}(\omega)} \leq c\left|u_{1}-\bar{g}\right|_{H^{1}(\omega)}+\|\bar{g}\|_{L^{2}(\omega)} \\
& \leq c\left|u_{1}\right|_{H^{1}(\omega)}+(1+c)\|\bar{g}\|_{H^{1}(\omega)},
\end{aligned}
$$


Combining all this yields

$$
\begin{aligned}
\sum_{i=1}^{2} \mu_{i}\left|u_{i}\right|_{H^{1}(\omega)}^{2} \leq c\left(\left(\left\|f_{1}\right\|_{L^{2}(\omega)}+\mu_{1}|\bar{g}|_{H^{1}(\omega)}\right)\left|u_{1}\right|_{H^{1}(\omega)}\right. & \\
& \left.+\left\|f_{2}\right\|_{L^{2}(\omega)}\left|u_{2}\right|_{H^{1}(\omega)}+\left\|f_{1}\right\|_{L^{2}(\omega)}\|\bar{g}\|_{H^{1}(\omega)}\right) .
\end{aligned}
$$

Using an appropriate Young's inequality together with (2.18) and (2.19) gives the estimate for the $\left\|u_{i}\right\|_{H^{1}(\omega)}$.

2) Finally, the estimate for $\|\lambda\|_{L^{2}(\omega)}$ is a direct consequence of (2.10) and the fact that $\lambda$ coincides with the functions $\lambda_{1}$ and $\lambda_{2}$ defined in (2.13).

Since $\lambda$ belongs to $L^{2}(\omega)$, the next proposition is easily derived from the regularity properties of the Laplace operator, see [8, Thm 3.2.1.2] and [8, §4.3] for instance.

Proposition 7. For any data $\left(f_{1}, f_{2}\right)$ in $L^{2}(\omega) \times L^{2}(\omega)$ and $g$ in $H_{+}^{s+\frac{1}{2}}(\partial \omega)$, the solution $\left(u_{1}, u_{2}, \lambda\right)$ of problem (2.4) belongs to $H^{s+1}(\omega) \times H^{s+1}(\omega) \times L^{2}(\omega)$, with

(i) $s=\frac{1}{2}$ in the general case,

(ii) $s=1$ when $\omega$ is convex or of class $\mathcal{C}^{1,1}$.

\section{The reduced discrete problem and its a priori analysis}

In view of Section 2, we first propose a discretization of problem (2.9) and perform its numerical analysis. In particular, we prove a priori error estimates.

From now on, we assume that $\omega$ is a polygon. Let $\left(\mathcal{T}_{h}\right)_{h}$ be a regular family of triangulations of $\omega$ (by triangles), in the usual sense that:

- For each $h, \bar{\omega}$ is the union of all elements of $\mathcal{T}_{h}$;

- The intersection of two different elements of $\mathcal{T}_{h}$, if not empty, is a vertex or a whole edge of both of them;

- The ratio of the diameter $h_{K}$ of any element $K$ of $\mathcal{T}_{h}$ to the diameter of its inscribed circle is smaller than a constant independent of $h$.

As usual, $h$ stands for the maximum of the diameters $h_{K}, K \in \mathcal{T}_{h}$. In what follows, $c, c^{\prime}, \ldots$, stand for generic constants which may vary from line to line but are always independent of $h$.

We will use the discrete spaces given as

$$
\mathbb{X}_{h}=\left\{v_{h} \in H^{1}(\omega) ; \forall K \in \mathcal{T}_{h},\left.v_{h}\right|_{K} \in \mathcal{P}_{1}(K)\right\}, \quad \mathbb{X}_{0 h}=\mathbb{X}_{h} \cap H_{0}^{1}(\omega),
$$

where $\mathcal{P}_{1}(K)$ denotes the space of restrictions to $K$ of affine functions.

Next, in order to take into account the nonhomogeneous boundary condition on $u_{1}$, we assume that the datum $g$ belongs to $H^{s+\frac{1}{2}}(\partial \omega)$ for some $s>0$. Thus, we define an approximation $g_{h}$ of $g$ by Lagrange interpolation: The function $g_{h}$ is affine on each edge $e$ of elements of $\mathcal{T}_{h}$ which is contained in $\partial \omega$ and equal to $g$ at each vertex of elements of $\mathcal{T}_{h}$ which belong to $\partial \omega$. Thus, we define the affine space

$$
\mathbb{X}_{g h}=\left\{v_{h} \in \mathbb{X}_{h} ; v_{h}=g_{h} \text { on } \partial \omega\right\}
$$


together with the convex set

$$
\mathcal{K}_{g h}=\left\{\left(v_{1 h}, v_{2 h}\right) \in \mathbb{X}_{g h} \times \mathbb{X}_{0 h} ; v_{1 h}-v_{2 h} \geq 0 \text { in } \omega\right\} .
$$

The reduced discrete problem is now derived from problem (2.9) by the Galerkin method. It reads:

Find $\left(u_{1 h}, u_{2 h}\right)$ in $\mathcal{K}_{g h}$ such that

$$
\begin{aligned}
\forall\left(v_{1 h}, v_{2 h}\right) \in \mathcal{K}_{g h}, \quad \sum_{i=1}^{2} \mu_{i} \int_{\omega}\left(\operatorname{grad} u_{i h}\right)(\boldsymbol{x}) \cdot\left(\operatorname{grad}\left(v_{i h}-u_{i h}\right)\right)(\boldsymbol{x}) \mathrm{d} \boldsymbol{x} \\
\geq \sum_{i=1}^{2}\left\langle f_{i}, v_{i h}-u_{i h}\right\rangle .
\end{aligned}
$$

The arguments for proving its well-posedness are exactly the same as for Proposition 3.

Proposition 8. For any data $\left(f_{1}, f_{2}\right)$ in $H^{-1}(\omega) \times H^{-1}(\omega)$ and $g$ in $H_{+}^{s+\frac{1}{2}}(\partial \omega), s>0$, problem (3.4) has a unique solution $\left(u_{1 h}, u_{2 h}\right)$ in $\mathcal{K}_{g h}$.

Proof: It follows from the previous assumptions that $g_{h}$ is affine on each edge of elements of $\mathcal{T}_{h}$ which is contained in $\partial \omega$ and nonnegative at the endpoints of this edge. So it is nonnegative on $\partial \omega$. Therefore, $\mathcal{K}_{g h}$ is a nonempty closed convex set. Since the ellipticity property (2.6) is still valid on $\mathbb{X}_{0 h} \times \mathbb{X}_{0 h}$, the result is easily derived from the Lions-Stampacchia theorem.

Theorem 9. Assume that the domain $\omega$ is convex, that the data $\left(f_{1}, f_{2}\right)$ belong to $L^{2}(\omega) \times L^{2}(\omega)$ and that the datum g belongs to $H_{+}^{\frac{3}{2}}(\partial \omega)$. Then, the following a priori error estimate holds between the solutions $\left(u_{1}, u_{2}\right)$ of problem $(2.9)$ and $\left(u_{1 h}, u_{2 h}\right)$ of problem (3.4)

$$
\left\|u_{1}-u_{1 h}\right\|_{H^{1}(\omega)}+\left\|u_{2}-u_{2 h}\right\|_{H^{1}(\omega)} \leq c h\left(\left\|f_{1}\right\|_{L^{2}(\omega)}+\left\|f_{2}\right\|_{L^{2}(\omega)}+\|g\|_{H^{\frac{3}{2}(\partial \omega)}}\right) .
$$

Proof: We establish this result in several steps, following the approach in [7].

1) Let $\boldsymbol{v}_{h}=\left(v_{1 h}, v_{2 h}\right)$ be any element of $\mathcal{K}_{g h}$. We derive by using problem (3.4) that

$$
\begin{aligned}
\sum_{i=1}^{2} \mu_{i} \mid u_{i h}- & \left.v_{i h}\right|_{H^{1}(\omega)} ^{2} \\
& \leq \sum_{i=1}^{2}\left(-\mu_{i} \int_{\omega}\left(\operatorname{grad} v_{i h}\right)(\boldsymbol{x}) \cdot\left(\operatorname{grad}\left(u_{i h}-v_{i h}\right)\right)(\boldsymbol{x}) \mathrm{d} \boldsymbol{x}+\left\langle f_{i}, u_{i h}-v_{i h}\right\rangle\right)
\end{aligned}
$$

whence, from problem (2.4),

$$
\begin{aligned}
& \sum_{i=1}^{2} \mu_{i}\left|u_{i h}-v_{i h}\right|_{H^{1}(\omega)}^{2} \\
& \quad \leq \sum_{i=1}^{2}\left(\mu_{i} \int_{\omega}\left(\operatorname{grad}\left(u_{i}-v_{i h}\right)\right)(\boldsymbol{x}) \cdot\left(\operatorname{grad}\left(u_{i h}-v_{i h}\right)\right)(\boldsymbol{x}) \mathrm{d} \boldsymbol{x}+b\left(\boldsymbol{u}_{h}-\boldsymbol{v}_{h}, \lambda\right)\right) .
\end{aligned}
$$


To evaluate this last term, we note from the definitions of the form $b(\cdot, \cdot)$, and also of $\Lambda$ and $\mathcal{K}_{g h}$ that

$$
b\left(\boldsymbol{u}_{h}, \lambda\right)=-\int_{\omega} \lambda(\boldsymbol{x})\left(u_{1 h}-u_{2 h}\right)(\boldsymbol{x}) \mathrm{d} \boldsymbol{x} \leq 0 .
$$

On the other hand, it follows from the second line of problem (2.4) by taking successively $\chi=0$ and $\chi=2 \lambda$ that $b(\boldsymbol{u}, \lambda)$ is zero. Thus, we obtain

$$
b\left(\boldsymbol{u}_{h}-\boldsymbol{v}_{h}, \lambda\right) \leq b\left(\boldsymbol{u}-\boldsymbol{v}_{h}, \lambda\right) \leq\left\|\boldsymbol{u}-\boldsymbol{v}_{h}\right\|_{L^{2}(\omega)^{2}}\|\lambda\|_{L^{2}(\omega)} .
$$

Going back to (3.6) and using appropriate Young's and triangle inequalities lead to

$$
\left|\boldsymbol{u}-\boldsymbol{u}_{h}\right|_{H^{1}(\omega)^{2}}^{2} \leq c\left(\left|\boldsymbol{u}-\boldsymbol{v}_{h}\right|_{H^{1}(\omega)^{2}}^{2}+\left\|\boldsymbol{u}-\boldsymbol{v}_{h}\right\|_{L^{2}(\omega)^{2}}\|\lambda\|_{L^{2}(\omega)}\right) .
$$

2) On the other hand, we derive from Proposition 4 , the formula $\lambda=(-1)^{i}\left(\Delta u_{i}+f_{i}\right)$, and the regularity properties of the Laplace operator [8, Thm 3.2.1.2] in a convex domain that $\boldsymbol{u}$ belongs to $H^{2}(\omega)^{2}$ (see also Proposition 7), together with

$$
\left\|u_{1}\right\|_{H^{2}(\omega)}+\left\|u_{2}\right\|_{H^{2}(\omega)}+\|\lambda\|_{L^{2}(\omega)} \leq c\left(\left\|f_{1}\right\|_{L^{2}(\omega)}+\left\|f_{2}\right\|_{L^{2}(\omega)}+\|g\|_{H^{\frac{3}{2}(\partial \omega)}}\right) .
$$

3) Let $\mathcal{I}_{h}$ denote the Lagrange interpolation operator at all vertices of elements of $\mathcal{T}_{h}$ with values in $\mathbb{X}_{h}$. The following approximation properties are standard [3, Lemmes IX.1.1 \& IX.1.2]

$$
\left\|v-\mathcal{I}_{h} v\right\|_{L^{2}(\omega)} \leq c h^{2}\|v\|_{H^{2}(\omega)}, \quad\left|v-\mathcal{I}_{h} v\right|_{H^{1}(\omega)} \leq c h\|v\|_{H^{2}(\omega)} .
$$

Furthermore, it is readily checked that, since $\left(u_{1}, u_{2}\right)$ belongs to $\mathcal{K}_{g}$,

- the trace of $\mathcal{I}_{h} u_{1}$ on $\partial \omega$ coincides with $g_{h}$, while the trace of $\mathcal{I}_{h} u_{2}$ is zero;

- on each element $K$ of $\mathcal{I}_{h}, \mathcal{I}_{h} u_{1}-\mathcal{I}_{h} u_{2}$ is affine and equal to $\left(u_{1}-u_{2}\right)(\boldsymbol{a})$ at each vertex $\boldsymbol{a}$ of $K$, hence is nonnegative on $K$.

Thus, $\left(\mathcal{I}_{h} u_{1}, \mathcal{I}_{h} u_{2}\right)$ belongs to $\mathcal{K}_{g h}$. Taking $\boldsymbol{v}_{h}$ equal to $\left(\mathcal{I}_{h} u_{1}, \mathcal{I}_{h} u_{2}\right)$, inserting (3.9) into (3.7), and using (3.8) give the desired estimate for $\left|\boldsymbol{u}-\boldsymbol{u}_{h}\right|_{H^{1}(\omega)^{2}}$.

4) Evaluating $\left\|u_{2}-u_{2 h}\right\|_{L^{2}(\omega)}$ thus follows from the Poincaré-Friedrichs inequality

$$
\left\|u_{2}-u_{2 h}\right\|_{L^{2}(\omega)} \leq c\left|u_{2}-u_{2 h}\right|_{H^{1}(\omega)} .
$$

To bound $\left\|u_{1}-u_{1 h}\right\|_{L^{2}(\omega)}$, we introduce a lifting $g^{*}$ of $g$ which now belongs to $H^{2}(\omega)$ and satisfies

$$
\left\|g^{*}\right\|_{H^{2}(\omega)} \leq c\|g\|_{H^{\frac{3}{2}}(\partial \omega)} .
$$

Thus, we derive by the same arguments as for (2.19)

$$
\left\|u_{1}-u_{1 h}\right\|_{L^{2}(\omega)} \leq c\left|u_{1}-u_{1 h}\right|_{H^{1}(\omega)}+(1+c)\left\|g^{*}-\mathcal{I}_{h} g^{*}\right\|_{H^{1}(\omega)} .
$$

Thus, using once more (3.9) gives the desired estimate.

Estimate (3.5) is fully optimal, since the discretization that we propose is of order 1.

Remark 10. When $\omega$ is not convex or for less smooth data, standard arguments yield estimate (3.5), with $h$ replaced by $h^{s}$ for $0<s \leq 1$. Optimal estimates can also be obtained when the mesh is refined in an exponential way near the re-entrant corners. 


\section{The full discrete problem and its a priori analysis}

We first show how to obtain a discrete action $\lambda_{h}$ from the finite element discrete displacements $u_{1 h}$ and $u_{2 h}$ by a local postprocessing. We next prove that the triple $\left(u_{1 h}, u_{2 h}, \lambda_{h}\right)$ in fact corresponds to a discretization of the full problem (2.4), where the displacements are discretized by finite elements on the given grid and the action by a finite volume-like scheme on a dual grid. This last property then allows us to obtain optimal a priori error estimates for the discrete action.

\subsection{Construction of the discrete action by local postprocessing}

We first describe the full discrete problem in a rather abstract way. Let $\mathcal{V}_{h}$ denote the set of vertices of elements of $\mathcal{T}_{h}$ which do not belong to $\partial \omega$. We thus introduce the Lagrange functions associated with the elements of $\mathcal{V}_{h}$ : For each $\boldsymbol{a}$ in $\mathcal{V}_{h}, \varphi_{\boldsymbol{a}}$ belongs to $\mathbb{X}_{0 h}$ and satisfies

$$
\varphi_{\boldsymbol{a}}(\boldsymbol{a})=1 \quad \text { and } \quad \forall \boldsymbol{a}^{\prime} \in \mathcal{V}_{h}, \boldsymbol{a}^{\prime} \neq \boldsymbol{a}, \quad \varphi_{\boldsymbol{a}}\left(\boldsymbol{a}^{\prime}\right)=0
$$

We also denote by $\mathcal{T}_{\boldsymbol{a}}$ the set of elements of $\mathcal{T}_{h}$ that contain $\boldsymbol{a}$ and by $h_{\boldsymbol{a}}$ the maximum of the diameters of the elements of $\mathcal{T}_{a}$. Finally, $\Delta_{a}$ stands for the support of $\varphi_{a}$.

To go further, we introduce a new set of nonnegative functions $\chi_{\boldsymbol{a}}, \boldsymbol{a} \in \mathcal{V}_{h}$, in $L^{2}(\omega)$, with support in a neighbourhood of $\boldsymbol{a}$, and define $\mathbb{Y}_{h}$ as the space spanned by these functions (so that the dimension of $\mathbb{Y}_{h}$ is equal to the dimension of $\mathbb{X}_{0 h}$ if the $\chi_{a}$ are linearly independent). Thus, it is readily checked that the set

$$
\Lambda_{h}=\left\{\rho_{h}=\sum_{\boldsymbol{a} \in \mathcal{V}_{h}} \rho_{\boldsymbol{a}} \chi_{\boldsymbol{a}} ; \rho_{\boldsymbol{a}} \geq 0\right\},
$$

is a convex cone contained in $\Lambda$. We also introduce a duality pairing between $\mathbb{Y}_{h}$ and $\mathbb{X}_{h}$ by

$$
\begin{aligned}
\forall \rho_{h}=\sum_{\boldsymbol{a} \in \mathcal{V}_{h}} \rho_{\boldsymbol{a}} \chi_{\boldsymbol{a}} \in \mathbb{Y}_{h}, \forall v_{h} & \in \mathbb{X}_{0 h}, \\
\left\langle\rho_{h}, v_{h}\right\rangle_{h} & =\sum_{\boldsymbol{a} \in \mathcal{V}_{h}} \rho_{\boldsymbol{a}} v_{h}(\boldsymbol{a}) \sum_{K \in \mathcal{T}_{\boldsymbol{a}}} \int_{K} \varphi_{\boldsymbol{a}}(\boldsymbol{x}) \mathrm{d} \boldsymbol{x} .
\end{aligned}
$$

Remark 11. It follows from the previous definition that, with the same notation,

$$
\left\langle\rho_{h}, \varphi_{\boldsymbol{a}}\right\rangle_{h}=\rho_{\boldsymbol{a}} \sum_{K \in \mathcal{T}_{\boldsymbol{a}}} \int_{K} \varphi_{\boldsymbol{a}}(\boldsymbol{x}) \mathrm{d} \boldsymbol{x} .
$$

So, since the $\varphi_{\boldsymbol{a}}, \boldsymbol{a} \in \mathcal{V}_{h}$, form a basis of $\mathbb{X}_{0 h}$, any function $\rho_{h}$ in $\mathbb{Y}_{h}$ is perfectly defined by the products $\left\langle\rho_{h}, v_{h}\right\rangle_{h}, v_{h} \in \mathbb{X}_{0 h}$.

In analogy with (2.14), we can now define functions $\lambda_{1 h}$ and $\lambda_{2 h}$ in $\mathbb{Y}_{h}$ by the equations, where $v_{1 h}$ and $v_{2 h}$ run through $\mathbb{X}_{0 h}$,

$$
\begin{aligned}
& \left\langle\lambda_{1 h}, v_{1 h}\right\rangle_{h}=\mu_{1} \int_{\omega}\left(\operatorname{grad} u_{1 h}\right)(\boldsymbol{x}) \cdot\left(\operatorname{grad} v_{1 h}\right)(\boldsymbol{x}) \mathrm{d} \boldsymbol{x}-\left\langle f_{1}, v_{1 h}\right\rangle, \\
& \left\langle\lambda_{2 h}, v_{2 h}\right\rangle_{h}=-\mu_{2} \int_{\omega}\left(\operatorname{grad} u_{2 h}\right)(\boldsymbol{x}) \cdot\left(\operatorname{grad} v_{2 h}\right)(\boldsymbol{x}) \mathrm{d} \boldsymbol{x}+\left\langle f_{2}, v_{2 h}\right\rangle .
\end{aligned}
$$


Note that the practical construction of $\lambda_{1 h}$ and $\lambda_{2 h}$ is fully local, since, owing to the previous remark, we can equivalently replace $v_{1 h}$ and $v_{2 h}$ by $\varphi_{\boldsymbol{a}}, \boldsymbol{a} \in \mathcal{V}_{h}$, in (4.5). Moreover, we have the following result.

Proposition 12. The functions $\lambda_{1 h}$ and $\lambda_{2 h}$ defined in (4.5) coincide.

Proof: Subtracting the second equation in (4.5) from the first one, replacing each $v_{i h}$ by $v_{i h}-u_{i h}$ for a pair $\left(v_{1 h}, v_{2 h}\right)$ in $\mathcal{K}_{g h}$, and using problem (3.4) yield that

$$
\left\langle\lambda_{1 h}, u_{1 h}-v_{1 h}\right\rangle_{h}-\left\langle\lambda_{2 h}, u_{2 h}-v_{2 h}\right\rangle_{h} \leq 0 .
$$

Let now $\varphi_{h}$ be any function in $\mathbb{X}_{0 h}$. Then, the pairs $\left(v_{1 h}=u_{1 h} \pm \varphi_{h}, v_{2 h}=u_{2 h} \pm \varphi_{h}\right)$ belong to $\mathcal{K}_{g h}$. Making this choice in (4.6) yields

$$
\left\langle\lambda_{1 h}-\lambda_{2 h}, \varphi_{h}\right\rangle_{h}=0
$$

It thus follows from Remark 11 that the functions $\lambda_{1 h}$ and $\lambda_{2 h}$ coincide.

In view of Proposition 12, we now write a discrete problem which, up to the replacement of the scalar product of $L^{2}(\omega)$ by the new duality pairing $\langle\cdot, \cdot\rangle_{h}$, is constructed from problem (2.4) by the Galerkin method. It reads:

Find $\left(u_{1 h}, u_{2 h}, \lambda_{h}\right)$ in $\mathbb{X}_{g h} \times \mathbb{X}_{0 h} \times \Lambda_{h}$ such that

$$
\begin{gathered}
\begin{aligned}
\forall\left(v_{1 h}, v_{2 h}\right) \in \mathbb{X}_{0 h} \times \mathbb{X}_{0 h}, \quad \sum_{i=1}^{2} \mu_{i} \int_{\omega}\left(\operatorname{grad} u_{i h}\right)(\boldsymbol{x}) \cdot\left(\operatorname{grad} v_{i h}\right)(\boldsymbol{x}) \mathrm{d} \boldsymbol{x} \\
\quad-\left\langle\lambda_{h}, v_{1 h}-v_{2 h}\right\rangle_{h}=\sum_{i=1}^{2}\left\langle f_{i}, v_{i h}\right\rangle, \\
\forall \chi_{h} \in \Lambda_{h}, \quad\left\langle\chi_{h}-\lambda_{h}, u_{1 h}-u_{2 h}\right\rangle_{h} \geq 0 .
\end{aligned}
\end{gathered}
$$

Note that this problem is not interesting for the implementation, but it is needed for the analysis. Indeed, we have the next result.

Lemma 13. For any solution $\left(u_{1 h}, u_{2 h}, \lambda_{h}\right)$ of problem (4.7), the pair $\left(u_{1 h}, u_{2 h}\right)$ is a solution of problem (3.4). Conversely, for any solution $\left(u_{1 h}, u_{2 h}\right)$ of problem (3.4), the function $\lambda_{h}=\lambda_{i h}$, $i=1,2$, defined by (4.5) gives rise to a solution $\left(u_{1 h}, u_{2 h}, \lambda_{h}\right)$ of problem (4.7).

Proof: It is performed in two steps.

1) Let $\left(u_{1 h}, u_{2 h}, \lambda_{h}\right)$ be a solution of problem (4.7). Replacing $\chi_{h}$ by $\chi_{h}+\lambda_{h}$ in the second line of (4.7) gives

$$
\forall \chi_{h} \in \Lambda_{h}, \quad\left\langle\chi_{h}, u_{1 h}-u_{2 h}\right\rangle_{h} \geq 0 .
$$

Thus, taking $\chi_{h}$ equal to $\chi_{\boldsymbol{a}}$ in the previous line yields that, for any $\boldsymbol{a}$ in $\mathcal{V}_{h},\left(u_{1 h}-u_{2 h}\right)(\boldsymbol{a})$ is nonnegative. On each vertex $\boldsymbol{a}$ of elements $K$ which belongs to $\partial \omega,\left(u_{1 h}-u_{2 h}\right)(\boldsymbol{a})$ is equal to 
$g(\boldsymbol{a})$, hence is nonnegative. Thus the pair $\left(u_{1 h}, u_{2 h}\right)$ belongs to $\mathcal{K}_{g h}$. On the other hand, using the definition of $\Lambda_{h}$, we derive that, for any $\left(v_{1 h}, v_{2 h}\right)$ in $\mathcal{K}_{g h}$,

$$
-\left\langle\lambda_{h}, v_{1 h}-v_{2 h}\right\rangle_{h} \leq 0 .
$$

By taking $\chi_{h}$ equal to 0 in the second line of (4.7), we also obtain that $\left\langle\lambda_{h}, u_{1 h}-u_{2 h}\right\rangle_{h} \leq 0$. Thus, by replacing each $v_{i h}$ in the first line of (4.7) by $v_{i h}-u_{i h}$ and using the two previous inequalities, we obtain (3.4).

2) Conversely, let $\left(u_{1 h}, u_{2 h}\right)$ be the solution of problem (3.4). Noting from Proposition 12 that the functions $\lambda_{1 h}$ and $\lambda_{2 h}$ defined in (4.5) coincide, we denote them by $\lambda_{h}$. We thus observe that the triple $\left(u_{1 h}, u_{2 h}, \lambda_{h}\right)$ satisfies the first line of problem (4.7). On the other hand, for each $\boldsymbol{a}$ in $\mathcal{V}_{h}$, using (4.6) with $\left(v_{1 h}=u_{1 h}+\varphi_{\boldsymbol{a}}, v_{2 h}=u_{2 h}\right)$ implies

$$
\left\langle\lambda_{h}, \varphi_{a}\right\rangle_{h} \geq 0
$$

It thus follows from (4.2) and (4.4) that $\lambda_{h}$ belongs to $\Lambda_{h}$. Finally, we now take in (4.6)

$$
v_{1 h}=u_{1 h}-\sum_{\boldsymbol{a} \in \mathcal{V}_{h}} u_{1 h}(\boldsymbol{a}) \varphi_{\boldsymbol{a}}, \quad v_{2 h}=0,
$$

so that $v_{1 h}$ is equal to $u_{1 h}$ on $\partial \omega$ but vanishes outside a small neighborhood of $\partial \omega$. As hinted in Remark 11, we have

$$
\left\langle\lambda_{h}, u_{1 h}\right\rangle_{h}=\left\langle\lambda_{h}, \sum_{\boldsymbol{a} \in \mathcal{V}_{h}} u_{1 h}(\boldsymbol{a}) \varphi_{\boldsymbol{a}}\right\rangle_{h}
$$

So, we derive

$$
-\left\langle\lambda_{h}, u_{1 h}-u_{2 h}\right\rangle_{h} \geq 0 \text {. }
$$

Using the definitions of $\Lambda_{h}$ and $\mathcal{K}_{g h}$ also yields that, for all $\chi_{h}$ in $\Lambda_{h}$,

$$
\left\langle\chi_{h}, u_{1 h}-u_{2 h}\right\rangle_{h} \geq 0
$$

Summing the last two inequalities gives the second line of (4.7). As a consequence, the triple $\left(u_{1 h}, u_{2 h}, \lambda_{h}\right)$ is a solution of (4.7).

Remark 14. An important property of problem (4.7) can be derived as follows. By taking $\chi_{h}$ successively equal to 0 and to $2 \lambda_{h}$ in the second line of this problem, we have

$$
\left\langle\lambda_{h}, u_{1 h}-u_{2 h}\right\rangle_{h}=0
$$

Thus, combining (4.3) with the definitions of $\mathcal{K}_{g h}$ and $\Lambda_{h}$ yields the discrete analogue of the complementary equality in the third line of problem (1.1):

$$
\forall \boldsymbol{a} \in \mathcal{V}_{h}, \quad \lambda_{\boldsymbol{a}}\left(u_{1 h}-u_{2 h}\right)(\boldsymbol{a})=0
$$

This proves the consistency of our approach. 
The well-posedness of problem (4.7) is now a direct consequence of Lemma 13.

Theorem 15. For any data $\left(f_{1}, f_{2}\right)$ in $L^{2}(\omega) \times L^{2}(\omega)$ and $g$ in $H_{+}^{s+\frac{1}{2}}(\partial \omega), s>0$, problem $(4.7)$ has a unique solution $\left(u_{1 h}, u_{2 h}, \lambda_{h}\right)$ in $\mathbb{X}_{g h} \times \mathbb{X}_{0 h} \times \Lambda_{h}$.

Remark 16. Let $\Pi_{h}$ be the operator defined from $L^{2}(\omega)$ into $\mathbb{Y}_{h}$ by

$$
\forall \boldsymbol{a} \in \mathcal{V}_{h}, \quad\left\langle\Pi_{h} \rho, \varphi_{\boldsymbol{a}}\right\rangle_{h}=\int_{\omega} \rho(\boldsymbol{x}) \varphi_{\boldsymbol{a}}(\boldsymbol{x}) \mathrm{d} \boldsymbol{x} .
$$

If

(i) the following bound holds

$$
\forall \rho_{h}=\sum_{\boldsymbol{a} \in \mathcal{V}_{h}} \rho_{\boldsymbol{a}} \chi_{\boldsymbol{a}} \in \mathbb{Y}_{h}, \quad\left\|\rho_{h}\right\|_{H^{-1}(\omega)} \leq c\left(\sum_{\boldsymbol{a} \in \mathcal{V}_{h}} \rho_{a}^{2} h_{a}^{4}\right)^{\frac{1}{2}},
$$

(ii) the following estimate holds for any function $\rho$ in $L^{2}(\omega)$

$$
\left\|\rho-\Pi_{h} \rho\right\|_{H^{-1}(\omega)} \leq c^{\prime} h\|\rho\|_{L^{2}(\omega)},
$$

and if moreover the assumptions of Theorem 9 are satisfied, standard arguments yield the following a priori error estimate for the discrete problem (4.7)

$$
\left\|\lambda-\lambda_{h}\right\|_{H^{-1}(\omega)} \leq c h\left(\left\|f_{1}\right\|_{L^{2}(\omega)}+\left\|f_{2}\right\|_{L^{2}(\omega)}+\|g\|_{H^{\frac{3}{2}(\partial \omega)}}\right) .
$$

Assumptions (4.10) and (4.11) are likely but seem rather difficult to establish for the examples that we have in mind.

\subsection{An example of full discretization}

We introduce the dual mesh of the triangulation $\mathcal{T}_{h}$, as standard for a finite volume approach. For each $\boldsymbol{a}$ in $\mathcal{V}_{h}$ and with each $K$ in $\mathcal{T}_{\boldsymbol{a}}$, we associate the quadrilateral with vertices $\boldsymbol{a}$, the midpoints of the two edges of $K$ that contain $\boldsymbol{a}$ and the barycentre of $K$. The union of these quadrilaterals when $K$ runs through $\mathcal{T}_{\boldsymbol{a}}$ is denoted by $D_{\boldsymbol{a}}$. This notation is illustrated in Figure 1 , and it can be observed that, if $\boldsymbol{a}$ is shared by $k$ elements of $\mathcal{T}_{h}, D_{\boldsymbol{a}}$ is a polygon with $2 k$ edges, and is always contained in $\Delta_{a}$.

The set of elements $D_{\boldsymbol{a}}, \boldsymbol{a} \in \mathcal{V}_{h}$, is denoted by $\mathcal{D}_{h}$. It can be noted that the elements of $\mathcal{D}_{h}$ form a partition of $\omega$ minus a "small" neighbourhood of $\partial \omega$. We denote this new domain by $\omega_{h}$.

From now on, we choose to take the functions $\chi_{a}$ introduced in Section 4.1 equal to the characteristic functions of the $D_{\boldsymbol{a}}$, i.e., the functions equal to 1 on $D_{\boldsymbol{a}}$ and to zero elsewhere.

To investigate the convergence of $\lambda_{h}$ to $\lambda$ when $h$ tends to zero, we introduce a $H(\operatorname{div})$ conforming reconstruction of the discrete diffusive flux, as previously used in [14] in the framework of a posteriori error estimation:

(i) The intersection of each $D_{\boldsymbol{a}}$ with a triangle $K$ of $\mathcal{T}_{\boldsymbol{a}}$ is the union of two triangles with vertex $\boldsymbol{a}$. 
We denote by $\mathcal{S}_{h}$ the triangulation of $\omega_{h}$ made by these triangles.

(ii) $H\left(\operatorname{div}, \omega_{h}\right)$ is the domain of the divergence operator in $L^{2}\left(\omega_{h}\right)^{2}$, namely the space of functions $s$ in $L^{2}\left(\omega_{h}\right)^{2}$ such that div $s$ belongs to $L^{2}\left(\omega_{h}\right)$.

(iii) Finally, we consider the space associated with Raviart-Thomas finite elements [12]

$$
\mathbb{Z}_{h}=\left\{\boldsymbol{s}_{h} \in H\left(\operatorname{div}, \omega_{h}\right) ; \forall \kappa \in \mathcal{S}_{h},\left.\boldsymbol{s}_{h}\right|_{\kappa} \in \mathcal{R} T(\kappa)\right\},
$$

where $\mathcal{R} T(\kappa)$ stands for the space of restrictions to $\kappa$ of polynomials of the form $\boldsymbol{c}+d \boldsymbol{x}, \boldsymbol{c} \in \mathbb{R}^{2}$, $d \in \mathbb{R}$. We recall from [12] that the linear forms: $\boldsymbol{s} \mapsto \int_{e}(\boldsymbol{s} \cdot \boldsymbol{n})(\tau) \mathrm{d} \tau$ where $\boldsymbol{n}$ is a unit normal vector to $e$ and $e$ runs through the edges of $\kappa$, are $\mathcal{R} T(\kappa)$-unisolvent and that the functions of $\mathbb{Z}_{h}$ have a constant normal trace on each edge of elements of $\mathcal{S}_{h}$.

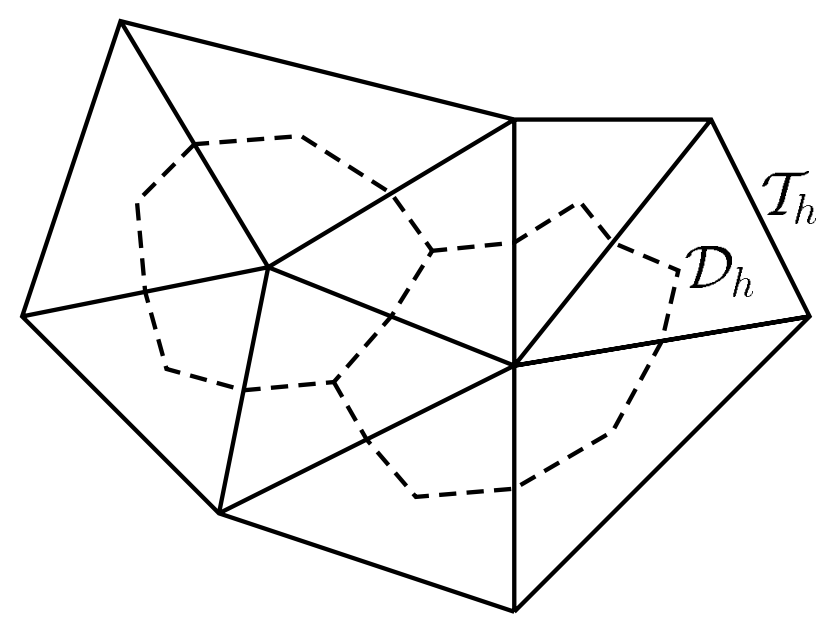

Figure 1: The triangulation $\mathcal{T}_{h}$ and its dual mesh $\mathcal{D}_{h}$

It follows from the properties stated above and the fact that the gradient of $\boldsymbol{u}_{i h}$ is constant on each element of $\mathcal{T}_{h}$ that, for $i=1$ and 2 , there exists a vector field $\boldsymbol{t}_{i h}$ in $\mathbb{Z}_{h}$ such that, for any edge $e$ of an element of $\mathcal{S}_{h}$ which is contained in one element $K$ of $\mathcal{T}_{h}$ (i.e., not contained in $\partial K$ ),

$$
\left.\left(\boldsymbol{t}_{i h} \cdot \boldsymbol{n}\right)\right|_{e}=-\mu_{i} \partial_{n}\left(\left.u_{i h}\right|_{K}\right) .
$$

The exact definition of the $\boldsymbol{t}_{i h}$ is made precise later on.

\subsection{A priori error estimates for the discrete action}

Establishing the error estimate between the exact and discrete actions relies on the functions $\boldsymbol{t}_{i h}$ introduced above. The main properties of each auxiliary function $\boldsymbol{t}_{i h}$ are stated in the next lemma. This requires the introduction of approximations $f_{i h}$ of the functions $f_{i}$ defined as follows: For $i=1$ and $2, f_{i h}$ is constant on each element $K$ of $\mathcal{T}_{h}$, defined by

$$
\forall K \in \mathcal{T}_{h},\left.\quad f_{i h}\right|_{K}=\frac{1}{\operatorname{meas}(K)} \int_{K} f_{i}(\boldsymbol{x}) \mathrm{d} \boldsymbol{x} .
$$


The main arguments for the proof of this lemma can be found in [1, Lemma 3]; we recall them only for completeness.

Lemma 17. The following equations are satisfied for $i=1$ and 2 and for any $\boldsymbol{a}$ in $\mathcal{V}_{h}$

$$
\begin{aligned}
& \int_{D_{\boldsymbol{a}}}\left(\operatorname{div} \boldsymbol{t}_{i h}+\mu_{i} \Delta u_{i}-(-1)^{i}\right.\left.\left(\lambda-\lambda_{h}\right)\right)(\boldsymbol{x}) \mathrm{d} \boldsymbol{x} \\
&=\int_{\Delta_{a}}\left(f_{i}-f_{i h}\right)(\boldsymbol{x}) \varphi_{\boldsymbol{a}}(\boldsymbol{x}) \mathrm{d} \boldsymbol{x}-\int_{D_{\boldsymbol{a}}}\left(f_{i}-f_{i h}\right)(\boldsymbol{x}) \mathrm{d} \boldsymbol{x} .
\end{aligned}
$$

Proof: We establish the result only for $i=1$ since the arguments for $i=2$ are exactly the same. This is performed in several steps. Let $\boldsymbol{a}$ be any vertex in $\mathcal{V}_{h}$.

1) Noting from Proposition 4 and Theorem 5 that $\Delta u_{1}$ and $\lambda$ belong to $L^{2}(\omega)$, we deduce from the first line of (1.1) that

$$
\int_{D_{\boldsymbol{a}}}\left(\mu_{1} \Delta u_{1}+\lambda\right)(\boldsymbol{x}) \mathrm{d} \boldsymbol{x}=-\int_{D_{\boldsymbol{a}}} f_{1}(\boldsymbol{x}) \mathrm{d} \boldsymbol{x} .
$$

2) We have

$$
\sum_{K \in \mathcal{T}_{\boldsymbol{a}}} \int_{K} f_{1 h}(\boldsymbol{x}) \varphi_{\boldsymbol{a}}(\boldsymbol{x}) \mathrm{d} \boldsymbol{x}=\left.\sum_{K \in \mathcal{T}_{\boldsymbol{a}}}\left(f_{1 h}\right)\right|_{K} \int_{K} \varphi_{\boldsymbol{a}}(\boldsymbol{x}) \mathrm{d} \boldsymbol{x}=\left.\sum_{K \in \mathcal{T}_{\boldsymbol{a}}}\left(f_{1 h}\right)\right|_{K} \frac{\operatorname{meas}(K)}{3} .
$$

Since the line joining a vertex of $K$ to the midpoint of its opposite edge divides $K$ into two equal parts, it is readily checked that $\frac{\operatorname{meas}(K)}{3}$ is equal to meas $\left(K \cap D_{\boldsymbol{a}}\right)$, whence

$$
\sum_{K \in \mathcal{T}_{a}} \int_{K} f_{1 h}(\boldsymbol{x}) \varphi_{\boldsymbol{a}}(\boldsymbol{x}) \mathrm{d} \boldsymbol{x}=\int_{D_{\boldsymbol{a}}} f_{1 h}(\boldsymbol{x}) \mathrm{d} \boldsymbol{x} .
$$

3) On the other hand, we have by integration by parts,

$$
\mu_{1} \sum_{K \in \mathcal{T}_{\boldsymbol{a}}} \int_{K}\left(\operatorname{grad} u_{1 h}\right)(\boldsymbol{x}) \cdot\left(\operatorname{grad} \varphi_{\boldsymbol{a}}\right)(\boldsymbol{x}) \mathrm{d} \boldsymbol{x}=\mu_{1} \sum_{K \in \mathcal{T}_{\boldsymbol{a}}} \int_{\partial K}\left(\left.\partial_{n} u_{1 h}\right|_{K}\right)(\tau) \varphi_{\boldsymbol{a}}(\tau) \mathrm{d} \tau
$$

where $\boldsymbol{n}$ here denotes the unit normal vector outward to $K$. We observe that $\partial K$ is the union of an edge contained in $\partial \Delta_{\boldsymbol{a}}$ where $\varphi_{\boldsymbol{a}}$ vanishes and of two edges $e_{1}$ and $e_{2}$; on these edges, $\left.\partial_{n} u_{1 h}\right|_{K}$ is constant and

$$
\int_{e_{j}} \varphi_{\boldsymbol{a}}(\tau) \mathrm{d} \tau=\frac{\operatorname{meas}\left(e_{j}\right)}{2}=\operatorname{meas}\left(e_{j} \cap D_{\boldsymbol{a}}\right)
$$

We also have

$$
0=\int_{K \cap D_{a}}\left(\Delta u_{1 h}\right)(\boldsymbol{x}) \mathrm{d} \boldsymbol{x}=\int_{\partial K \cap D_{a}}\left(\left.\partial_{n} u_{1 h}\right|_{K}\right)(\tau) \mathrm{d} \tau+\int_{K \cap \partial D_{a}}\left(\left.\partial_{n} u_{1 h}\right|_{K}\right)(\tau) \mathrm{d} \tau,
$$

whence

$$
\begin{aligned}
\mu_{1} \sum_{K \in \mathcal{T}_{\boldsymbol{a}}} \int_{K}\left(\operatorname{grad} u_{1 h}\right)(\boldsymbol{x}) \cdot\left(\operatorname{grad} \varphi_{\boldsymbol{a}}\right)(\boldsymbol{x}) \mathrm{d} \boldsymbol{x} & \\
= & -\mu_{1} \int_{\partial D_{\boldsymbol{a}}}\left(\partial_{n} u_{1 h}\right)(\tau) \mathrm{d} \tau=\int_{\partial D_{\boldsymbol{a}}}\left(\boldsymbol{t}_{1 h} \cdot \boldsymbol{n}\right)(\tau) \mathrm{d} \tau .
\end{aligned}
$$


This in turn yields

$$
\mu_{1} \sum_{K \in \mathcal{T}_{\boldsymbol{a}}} \int_{K}\left(\operatorname{grad} u_{1 h}\right)(\boldsymbol{x}) \cdot\left(\operatorname{grad} \varphi_{\boldsymbol{a}}\right)(\boldsymbol{x}) \mathrm{d} \boldsymbol{x}=\int_{D_{\boldsymbol{a}}}\left(\operatorname{div} \boldsymbol{t}_{1 h}\right)(\boldsymbol{x}) \mathrm{d} \boldsymbol{x} .
$$

4) By taking $v_{1 h}$ equal to $\varphi_{a}$ and $v_{2 h}$ equal to 0 in (4.7) and using (4.4), we derive

$$
\lambda_{\boldsymbol{a}} \operatorname{meas}\left(D_{\boldsymbol{a}}\right)=\mu_{1} \int_{\omega}\left(\operatorname{grad} u_{1 h}\right)(\boldsymbol{x}) \cdot\left(\operatorname{grad} \varphi_{\boldsymbol{a}}\right)(\boldsymbol{x}) \mathrm{d} \boldsymbol{x}-\int_{\omega} f_{1}(\boldsymbol{x}) \varphi_{\boldsymbol{a}}(\boldsymbol{x}) \mathrm{d} \boldsymbol{x} .
$$

Since the function $\varphi_{a}$ has its support equal to $\Delta_{a}$, inserting (4.17) and (4.18) into this equation yields

$$
\int_{D_{\boldsymbol{a}}} \lambda_{h}(\boldsymbol{x}) \mathrm{d} \boldsymbol{x}=\int_{D_{\boldsymbol{a}}}\left(\operatorname{div} \boldsymbol{t}_{1 h}-f_{1 h}\right)(\boldsymbol{x}) \mathrm{d} \boldsymbol{x}-\int_{\Delta_{\boldsymbol{a}}}\left(f_{1}-f_{1 h}\right)(\boldsymbol{x}) \varphi_{\boldsymbol{a}}(\boldsymbol{x}) \mathrm{d} \boldsymbol{x} .
$$

Combining this with (4.16) yields the desired result.

Corollary 18. If the data $\left(f_{1}, f_{2}\right)$ belong to $L^{2}(\omega) \times L^{2}(\omega)$, the following estimates are satisfied for $i=1$ and 2 :

$$
\begin{aligned}
\left\|\operatorname{div} \boldsymbol{t}_{i h}+\mu_{i} \Delta u_{i}-(-1)^{i}\left(\lambda-\lambda_{h}\right)\right\|_{H^{-1}(\omega)} \\
\quad \leq c h\left(\left\|f_{1}\right\|_{L^{2}(\omega)}+\left\|f_{2}\right\|_{L^{2}(\omega)}+\left\|\operatorname{div} \boldsymbol{t}_{1 h}\right\|_{L^{2}\left(\omega_{h}\right)}+\left\|\operatorname{div} \boldsymbol{t}_{2 h}\right\|_{L^{2}\left(\omega_{h}\right)}\right) .
\end{aligned}
$$

Proof: Denoting by $\rho$ the scalar function div $\boldsymbol{t}_{i h}+\mu_{i} \Delta u_{i}-(-1)^{i}\left(\lambda-\lambda_{h}\right)$, we have

$$
\|\rho\|_{H^{-1}(\omega)}=\sup _{w \in H_{0}^{1}(\omega)} \frac{\int_{\omega} \rho(\boldsymbol{x}) w(\boldsymbol{x}) \mathrm{d} \boldsymbol{x}}{\|w\|_{H^{1}(\omega)}} .
$$

In what follows, we write the integral on $\omega$ as a sum on two subdomains:

$$
\int_{\omega} \rho(\boldsymbol{x}) w(\boldsymbol{x}) \mathrm{d} \boldsymbol{x}=\int_{\omega \backslash \omega_{h}} \rho(\boldsymbol{x}) w(\boldsymbol{x}) \mathrm{d} \boldsymbol{x}+\int_{\omega_{h}} \rho(\boldsymbol{x}) w(\boldsymbol{x}) \mathrm{d} \boldsymbol{x} .
$$

1) Using the expansion $\lambda_{h}=\sum_{\boldsymbol{a} \in \mathcal{V}_{h}} \lambda_{\boldsymbol{a}} \chi_{\boldsymbol{a}}$ and noting that the measure of $D_{\boldsymbol{a}}$ (and also that of $\Delta_{a}$ ) is bounded from above and below by a constant times $h_{a}^{2}$, we derive from (4.15) that

$$
\left|\lambda_{\boldsymbol{a}}\right| h_{\boldsymbol{a}}^{2} \leq c h_{\boldsymbol{a}}\left(\left\|f_{i}-f_{i h}\right\|_{L^{2}\left(\Delta_{\boldsymbol{a}}\right)}+\left\|\operatorname{div} \boldsymbol{t}_{i h}\right\|_{L^{2}\left(D_{\boldsymbol{a}}\right)}+\left\|\Delta u_{i}\right\|_{L^{2}\left(D_{\boldsymbol{a}}\right)}+\|\lambda\|_{L^{2}\left(D_{\boldsymbol{a}}\right)}\right) .
$$

Thanks to the estimate

$$
\left\|\lambda_{h}\right\|_{L^{2}\left(\omega_{h}\right)}^{2} \leq c \sum_{\boldsymbol{a} \in \mathcal{V}_{h}} \lambda_{\boldsymbol{a}}^{2} h_{\boldsymbol{a}}^{2}
$$

we obtain

$$
\left\|\lambda_{h}\right\|_{L^{2}\left(\omega_{h}\right)} \leq c\left(\left\|f_{i}-f_{i h}\right\|_{L^{2}(\omega)}+\left\|\operatorname{div} \boldsymbol{t}_{i h}\right\|_{L^{2}\left(\omega_{h}\right)}+\left\|\Delta u_{i}\right\|_{L^{2}(\omega)}+\|\lambda\|_{L^{2}(\omega)}\right) .
$$


It follows from Proposition 4 and Corollary 6 that all terms in the right-hand side of this inequality are bounded. This yields the estimate

$$
\|\rho\|_{L^{2}(\omega)} \leq c\left(\left\|f_{1}\right\|_{L^{2}(\omega)}+\left\|f_{2}\right\|_{L^{2}(\omega)}+\left\|\operatorname{div} \boldsymbol{t}_{1 h}\right\|_{L^{2}\left(\omega_{h}\right)}+\left\|\operatorname{div} \boldsymbol{t}_{2 h}\right\|_{L^{2}\left(\omega_{h}\right)}\right) .
$$

2) It can be noted that $\omega \backslash \omega_{h}$ is contained in the union of elements $K$ of $\mathcal{T}_{h}$ which have at least one edge contained in $\partial \omega$. On each of these triangles, we derive by applying the Poincaré-Friedrichs inequality that, for any $w$ in $H_{0}^{1}(\omega)$,

$$
\|w\|_{L^{2}(K)} \leq c h_{K}|w|_{H^{1}(K)}
$$

As a consequence, we have

$$
\left|\int_{\omega \backslash \omega_{h}} \rho(\boldsymbol{x}) w(\boldsymbol{x}) \mathrm{d} \boldsymbol{x}\right| \leq c h\|\rho\|_{L^{2}(\omega)}\|w\|_{H^{1}(\omega)} .
$$

3) Let $w_{h}$ be the function equal to $w_{\boldsymbol{a}}$ on each $D_{\boldsymbol{a}}$, with

$$
w_{\boldsymbol{a}}=\frac{1}{\operatorname{meas}\left(D_{\boldsymbol{a}}\right)} \int_{D_{a}} w(\boldsymbol{x}) \mathrm{d} \boldsymbol{x} .
$$

Denoting by $\tilde{w}_{h}$ the function $\sum_{\boldsymbol{a} \in \mathcal{V}_{h}} w_{\boldsymbol{a}} \varphi_{\boldsymbol{a}}$, we derive from (4.15) that

$$
\begin{aligned}
\int_{\omega_{h}} \rho(\boldsymbol{x}) w(\boldsymbol{x}) \mathrm{d} \boldsymbol{x} & =\int_{\omega_{h}} \rho(\boldsymbol{x})\left(w-w_{h}\right)(\boldsymbol{x}) \mathrm{d} \boldsymbol{x}+\int_{\omega}\left(f_{i}-f_{i h}\right)(\boldsymbol{x}) \tilde{w}_{h}(\boldsymbol{x}) \mathrm{d} \boldsymbol{x} \\
& -\int_{\omega_{h}}\left(f_{i}-f_{i h}\right)(\boldsymbol{x}) w(\boldsymbol{x}) \mathrm{d} \boldsymbol{x}+\int_{\omega_{h}}\left(f_{i}-f_{i h}\right)(\boldsymbol{x})\left(w-w_{h}\right)(\boldsymbol{x}) \mathrm{d} \boldsymbol{x}
\end{aligned}
$$

It is well-known $[3, \S I X .3]$ that

$$
\left\|w-w_{h}\right\|_{L^{2}\left(\omega_{h}\right)} \leq c h\|w\|_{H^{1}\left(\omega_{h}\right)}, \quad\left\|\tilde{w}_{h}\right\|_{H^{1}(\omega)} \leq c\|w\|_{H^{1}(\omega)} .
$$

On the other hand, since the $f_{i h}$ are the images of the $f_{i}$ by the orthogonal projection operator from $L^{2}(\omega)$ onto piecewise constant functions, a standard duality argument yields

$$
\left\|f_{i}-f_{i h}\right\|_{H^{-1}(\omega)} \leq c h\left\|f_{i}\right\|_{L^{2}(\omega)}
$$

Combining all this leads to

$$
\left|\int_{\omega_{h}} \rho(\boldsymbol{x}) w(\boldsymbol{x}) \mathrm{d} \boldsymbol{x}\right| \leq c h\left(\|\rho\|_{L^{2}(\omega)}+\left\|f_{1}\right\|_{L^{2}(\omega)}+\left\|f_{2}\right\|_{L^{2}(\omega)}\right)\|w\|_{H^{1}(\omega)} .
$$

Inserting (4.23) and (4.26) into (4.21) and finally using (4.20) and (4.22) give the desired result.

The arguments for the next lemma are nearly the same as above. 
Lemma 19. If the assumptions of Theorem 9 are satisfied, the following estimates hold for $i=1$ and 2:

$$
\begin{aligned}
\left\|\operatorname{div} \boldsymbol{t}_{i h}+\mu_{i} \Delta u_{i}\right\|_{H^{-1}(\omega)} \leq c h\left(\left\|f_{1}\right\|_{L^{2}(\omega)}\right. & +\left\|f_{2}\right\|_{L^{2}(\omega)}+\|g\|_{H^{\frac{3}{2}(\partial \omega)}} \\
& \left.+\left\|\operatorname{div} \boldsymbol{t}_{1 h}\right\|_{L^{2}\left(\omega_{h}\right)}+\left\|\operatorname{div} \boldsymbol{t}_{2 h}\right\|_{L^{2}\left(\omega_{h}\right)}\right) .
\end{aligned}
$$

Proof: We now denote by $\rho$ the function $\operatorname{div} \boldsymbol{t}_{i h}+\mu_{i} \Delta u_{i}$ and use once more (4.20). The first term in the right-hand side of (4.21) is evaluated as in (4.23). To bound the second term, we use the same approximations $w_{h}$ and $\tilde{w}_{h}$ as previously and deduce from (4.18) that

$$
\begin{aligned}
\int_{\omega_{h}} \rho(\boldsymbol{x}) w(\boldsymbol{x}) \mathrm{d} \boldsymbol{x} & =\int_{\omega_{h}} \rho(\boldsymbol{x})\left(w-w_{h}\right)(\boldsymbol{x}) \mathrm{d} \boldsymbol{x} \\
& +\mu_{i} \int_{\omega}\left(\operatorname{grad} u_{i h}\right)(\boldsymbol{x}) \cdot\left(\operatorname{grad} \tilde{w}_{h}\right)(\boldsymbol{x}) \mathrm{d} \boldsymbol{x}+\mu_{i} \int_{\omega_{h}}\left(\Delta u_{i}\right)(\boldsymbol{x}) w_{h}(\boldsymbol{x}) \mathrm{d} \boldsymbol{x} .
\end{aligned}
$$

By integration by parts, this yields

$$
\begin{aligned}
& \int_{\omega_{h}} \rho(\boldsymbol{x}) w(\boldsymbol{x}) \mathrm{d} \boldsymbol{x}=\int_{\omega_{h}} \rho(\boldsymbol{x})\left(w-w_{h}\right)(\boldsymbol{x}) \mathrm{d} \boldsymbol{x} \\
& -\mu_{i} \int_{\omega}\left(\operatorname{grad}\left(u_{i}-u_{i h}\right)\right)(\boldsymbol{x}) \cdot\left(\operatorname{grad} \tilde{w}_{h}\right)(\boldsymbol{x}) \mathrm{d} \boldsymbol{x}+\mu_{i} \int_{\omega}\left(\Delta u_{i}\right)(\boldsymbol{x})\left(w-\tilde{w}_{h}\right)(\boldsymbol{x}) \mathrm{d} \boldsymbol{x} \\
& \quad-\mu_{i} \int_{\omega_{h}}\left(\Delta u_{i}\right)(\boldsymbol{x})\left(w-w_{h}\right)(\boldsymbol{x}) \mathrm{d} \boldsymbol{x}-\mu_{i} \int_{\omega \backslash \omega_{h}}\left(\Delta u_{i}\right)(\boldsymbol{x}) w(\boldsymbol{x}) \mathrm{d} \boldsymbol{x} .
\end{aligned}
$$

All these terms are evaluated from (4.24) (and an extension of it), Theorem 9 and the same arguments as for (4.22).

In view of Corollary 18 and Lemma 19, we need to check that both quantities $\left\|\operatorname{div} \boldsymbol{t}_{i h}\right\|_{L^{2}\left(\omega_{h}\right)}$ are bounded as a function of the data.

Lemma 20. Assume that the domain $\omega$ is convex and that there exists a constant $\sigma$ independent of $h$ such that

$$
\forall K \in \mathcal{T}_{h}, \quad h_{K} \geq \sigma h .
$$

For $i=1$ and 2 , there exists a function $\boldsymbol{t}_{i h}$ in $\mathbb{Z}_{h}$ satisfying (4.13) and such that

$$
\left\|\operatorname{div} \boldsymbol{t}_{i h}\right\|_{L^{2}\left(\omega_{h}\right)} \leq c\left(\left\|f_{1}\right\|_{L^{2}(\omega)}+\left\|f_{2}\right\|_{L^{2}(\omega)}+\|g\|_{H^{\frac{3}{2}(\partial \omega)}}\right) .
$$

Proof: We set: $\boldsymbol{t}_{i}=-\mu_{i}$ grad $u_{i}$ and introduce the so-called Raviart-Thomas operator $\pi_{h}$ [12]: For each sufficiently smooth vector field $\varphi, \pi_{h} \varphi$ belongs to $\mathbb{Z}_{h}$ and satisfies for any edge $e$ of an element of $\mathcal{S}_{h}$

$$
\int_{e}\left(\pi_{h} \boldsymbol{\varphi} \cdot \boldsymbol{n}\right)(\tau) \mathrm{d} \tau=\int_{e}(\boldsymbol{\varphi} \cdot \boldsymbol{n})(\tau) \mathrm{d} \tau
$$

Thus, we have

$$
\left\|\operatorname{div} \boldsymbol{t}_{i h}\right\|_{L^{2}\left(\omega_{h}\right)} \leq\left\|\operatorname{div} \pi_{h} \boldsymbol{t}_{i}\right\|_{L^{2}\left(\omega_{h}\right)}+\left\|\operatorname{div}\left(\boldsymbol{t}_{i h}-\pi_{h} \boldsymbol{t}_{i}\right)\right\|_{L^{2}\left(\omega_{h}\right)} .
$$


As well-known, div $\pi_{h} \boldsymbol{t}_{i}$ is the orthogonal projection of div $\boldsymbol{t}_{i}=-\mu_{i} \Delta u_{i}$ onto piecewise constant functions for the scalar product of $L^{2}(\Omega)$, so that the first term in the previous sum is bounded as desired, see Proposition 4. On the other hand, there exists a unique function $\boldsymbol{t}_{i h}$ in $\mathbb{Z}_{h}$ which satisfies (4.13) and such that, for any edge $e$ of an element of $\mathcal{S}_{h}$ which is contained in the boundary of an element $K$ of $\mathcal{T}_{h}$,

$$
\left.\left(\boldsymbol{t}_{i h} \cdot \boldsymbol{n}\right)\right|_{e}=-\mu_{i} \frac{1}{\operatorname{meas}(e)} \int_{e}\left(\partial_{n} u_{i}\right)(\tau) \mathrm{d} \tau
$$

(note that the previous integral makes sense; indeed, since $\omega$ is convex, $u_{i}$ belongs to $H^{2}(\omega)$ ). Thus, $\left(\boldsymbol{t}_{i h}-\pi_{h} \boldsymbol{t}_{i}\right) \cdot \boldsymbol{n}$ vanishes on such edges. Next, since $\operatorname{div}\left(\boldsymbol{t}_{i h}-\pi_{h} \boldsymbol{t}_{i}\right)$ is constant on each element $\kappa$ of $\mathcal{S}_{h}$, we note that

$$
\begin{aligned}
\left\|\operatorname{div}\left(\boldsymbol{t}_{i h}-\pi_{h} \boldsymbol{t}_{i}\right)\right\|_{L^{2}(\kappa)}^{2} & =\left.\int_{\partial \kappa}\left(\operatorname{div}\left(\boldsymbol{t}_{i h}-\pi_{h} \boldsymbol{t}_{i}\right)\right)\right|_{\kappa}\left(\left(\boldsymbol{t}_{i h}-\pi_{h} \boldsymbol{t}_{i}\right) \cdot \boldsymbol{n}\right)(\tau) \mathrm{d} \tau \\
& \leq c h_{K}^{-1}\left\|\operatorname{div}\left(\boldsymbol{t}_{i h}-\pi_{h} \boldsymbol{t}_{i}\right)\right\|_{L^{2}(\kappa)}\left|\int_{\partial \kappa}\left(\left(\boldsymbol{t}_{i h}-\pi_{h} \boldsymbol{t}_{i}\right) \cdot \boldsymbol{n}\right)(\tau) \mathrm{d} \tau\right| .
\end{aligned}
$$

Let now $\mathcal{E}_{\kappa}$ denote the set of the two edges of $\kappa$ which are inside an element $K$ of $\mathcal{T}_{h}$. We deduce from the previous lines that

$$
\left\|\operatorname{div}\left(\boldsymbol{t}_{i h}-\pi_{h} \boldsymbol{t}_{i}\right)\right\|_{L^{2}(\kappa)} \leq c h_{\kappa}^{-1} \sum_{e \in \mathcal{E}_{\kappa}}\left\|\partial_{n}\left(u_{i}-u_{i h}\right)\right\|_{L^{1}(e)} .
$$

To go further, we introduce the Lagrange interpolate $u_{i h}^{*}$ of $u_{i}$ on $\omega_{h}$, with values in the space

$$
\mathbb{X}_{h}^{*}=\left\{v_{h} \in H^{1}\left(\omega_{h}\right) ; \forall \kappa \in \mathcal{S}_{h},\left.v_{h}\right|_{\kappa} \in \mathcal{P}_{1}(\kappa)\right\},
$$

and we use the triangle inequality

$$
\left\|\partial_{n}\left(u_{i}-u_{i h}\right)\right\|_{L^{1}(e)} \leq\left\|\partial_{n}\left(u_{i}-\left.u_{i h}^{*}\right|_{\kappa}\right)\right\|_{L^{1}(e)}+\left\|\partial_{n}\left(\left.u_{i h}^{*}\right|_{\kappa}-u_{i h}\right)\right\|_{L^{1}(e)} .
$$

Next, by going to the reference element (with obvious notation) and noting that the Lagrange interpolation operator introduced above preserves the polynomials in $\mathcal{P}_{1}(\kappa)$, we derive

$$
\left\|\partial_{n}\left(u_{i}-\left.u_{i h}^{*}\right|_{\kappa}\right)\right\|_{L^{1}(e)} \leq c\left\|\operatorname{grad}\left(\hat{u}_{i}-\hat{u}_{i h}^{*}\right)\right\|_{L^{1}(\hat{e})^{2}} \leq c^{\prime}\left\|\hat{u}_{i}-\hat{u}_{i h}^{*}\right\|_{H^{2}(\hat{\kappa})} \leq c^{\prime \prime}\left|\hat{u}_{i}\right|_{H^{2}(\hat{\kappa})},
$$

whence

$$
\left\|\partial_{n}\left(u_{i}-\left.u_{i h}^{*}\right|_{\kappa}\right)\right\|_{L^{1}(e)} \leq c h_{\kappa}\left\|u_{i}\right\|_{H^{2}(\kappa)} .
$$

To evaluate the second term in the right-hand member of (4.31), we note that, for any function $\varphi$ in $\mathcal{P}_{0}(\kappa)$,

$$
\|\varphi\|_{L^{1}(e)} \leq c h_{\kappa}\|\hat{\varphi}\|_{L^{1}(\hat{e})} \leq c^{\prime} h_{\kappa}\|\hat{\varphi}\|_{L^{2}(\hat{\kappa})} \leq c^{\prime \prime}\|\varphi\|_{L^{2}(\kappa)} .
$$

Since $\left|\partial_{n}\left(\left.u_{i h}^{*}\right|_{\kappa}-u_{i h}\right)\right|$ is smaller than $\left|\operatorname{grad}\left(u_{i h}^{*}-u_{i h}\right)\right|$, this yields

$$
\left\|\partial_{n}\left(\left.u_{i h}^{*}\right|_{\kappa}-u_{i h}\right)\right\|_{L^{1}(e)} \leq c\left(\left|u_{i}-u_{i h}^{*}\right|_{H^{1}(\kappa)}+\left|u_{i}-u_{i h}\right|_{H^{1}(\kappa)}\right) .
$$


The approximation properties of the Lagrange interpolation operator finally give

$$
\left\|\partial_{n}\left(\left.u_{i h}^{*}\right|_{\kappa}-u_{i h}\right)\right\|_{L^{1}(e)} \leq c\left(h_{\kappa}\left\|u_{i}\right\|_{H^{2}(\kappa)}+\left|u_{i}-u_{i h}\right|_{H^{1}(\kappa)}\right) .
$$

By inserting (4.32) and (4.33) into (4.31) and using (4.30), we obtain

$$
\left\|\operatorname{div}\left(\boldsymbol{t}_{i h}-\pi_{h} \boldsymbol{t}_{i}\right)\right\|_{L^{2}(\kappa)} \leq c\left(\left\|u_{i}\right\|_{H^{2}(\kappa)}+h_{\kappa}^{-1}\left|u_{i}-u_{i h}\right|_{H^{1}(\kappa)}\right) .
$$

We conclude by summing the square of this inequality on all $\kappa$ in $\mathcal{S}_{h}$, using estimate (3.5) and finally assumption (4.28).

The final a priori estimate is now a direct consequence of Corollary 18 and Lemmas 19 and 20.

Theorem 21. If the assumptions of Theorem 9 are satisfied together with assumption (4.28), the following a priori error estimate holds between the solutions $\left(u_{1}, u_{2}, \lambda\right)$ of problem (2.4) and $\left(u_{1 h}, u_{2 h}, \lambda_{h}\right)$ of problem $(4.7)$

$$
\left\|\lambda-\lambda_{h}\right\|_{H^{-1}(\omega)} \leq c h\left(\left\|f_{1}\right\|_{L^{2}(\omega)}+\left\|f_{2}\right\|_{L^{2}(\omega)}+\|g\|_{H^{\frac{3}{2}(\partial \omega)}}\right) .
$$

Estimate (4.34) is fully optimal (and should hold with weaker norms of the data, however we have no applications for that). However, assumption (4.28) is very restrictive in the context of mesh adaptivity. Fortunately, if it is replaced by the more realistic one

$$
\forall K \in \mathcal{T}_{h}, \quad h_{K} \geq \sigma h^{1+\alpha},
$$

for a real number $\alpha, 0<\alpha<1$, exactly the same arguments as previously yield a convergence of $\left\|\lambda-\lambda_{h}\right\|_{H^{-1}(\omega)}$ of order $h^{1-\alpha}$.

Remark 22. By replacing (4.20) by

$$
\|\rho\|_{L^{2}(\omega)}=\sup _{w \in L^{2}(\omega)} \frac{\int_{\omega} \rho(\boldsymbol{x}) w(\boldsymbol{x}) \mathrm{d} \boldsymbol{x}}{\|w\|_{L^{2}(\omega)}},
$$

and using the same arguments as previously, we can also prove that, if the assumptions of Theorem 21 are satisfied,

$$
\lim _{h \rightarrow 0}\left\|\lambda-\lambda_{h}\right\|_{L^{2}(\omega)}=0 .
$$

With further regularity assumptions on the data, an explicit estimate of $\left\|\lambda-\lambda_{h}\right\|_{L^{2}(\omega)}$ as a function of $h$ can also be derived: For instance, when $f_{1 \mid K}$ and $f_{2 \mid K}$ belong to $H^{1}(K)$ for all $K$ in $\mathcal{T}_{h}$, this estimate is of order $h$.

It follows from the previous results that the discretization that we propose is very efficient. It is also of low cost since the total number of degrees of freedom is equal to three times the cardinality of $\mathcal{V}_{h}$. Moreover, the reduced problem (3.4) provides a natural algorithm for uncoupling the unknowns and, once its solution $\left(u_{1 h}, u_{2 h}\right)$ is known, computing $\lambda_{h}$ consists in solving a linear system with diagonal matrix. 


\section{References}

[1] R.E. Bank, D.J. Rose. Some error estimates for the box method. SIAM J. Numer. Anal., 24 (1987), 777-787.

[2] F. Ben Belgacem, C. Bernardi, A. Blouza, M. Vohralík. A finite element discretization of the contact between two membranes. Math. Model. Numer. Anal. (2009) DOI: 10.1051/m2an:2008041.

[3] C. Bernardi, Y. Maday, F. Rapetti. Discrétisations variationnelles de problèmes aux limites elliptiques. Collection "Mathématiques et Applications" 45, Springer-Verlag, Berlin, 2004.

[4] H. Brezis, G. Stampacchia. Sur la régularité de la solution d'inéquations elliptiques. Bull. Soc. Math. France, 96 (1968), 153-180.

[5] F. Brezzi, W.W. Hager, P.-A. Raviart. Error estimates for the finite element solution of variational inequalities. II. Mixed methods. Numer. Math., 31 (1978/79), 1-16.

[6] P.G. Ciarlet. The Finite Element Method for Elliptic Problems. Classics in Applied Mathematics 40, Society for Industrial and Applied Mathematics, 2002.

[7] R.S. Falk. Error estimates for the approximation of a class of variational inequalities. Math. Comput., 28 (1974), 963-971.

[8] P. Grisvard. Elliptic Problems in Nonsmooth Domains. Pitman, 1985.

[9] J. Haslinger, I. Hlaváček, J. Nečas. Numerical methods for unilateral problems in solid mechanics. In: Handbook of Numerical Analysis, Vol. IV, P.G. Ciarlet \& J.-L. Lions eds. NorthHolland, Amsterdam (1996), pp. 313-485.

[10] N. Kikuchi, J. T. Oden. Contact Problems in Elasticity: A Study of Variational Inequalities and Finite Element Methods. Studies in Applied and Numerical Mathematics, Society for Industrial and Applied Mathematics, 1988.

[11] J.-L. Lions, G. Stampacchia. Variational inequalities. Comm. Pure and Appl. Math., 20 (1967), 493-519.

[12] P.-A. Raviart, J.-M. Thomas. A mixed finite element method for second order elliptic problems. In: Mathematical Aspects of Finite Element Methods, Lecture Notes in Mathematics, 606, Springer, 1977, pp. 292-315.

[13] L. Slimane, A. Bendali, P. Laborde. Mixed formulations for a class of variational inequalities. Math. Model. Numer. Anal., 38 (2004), 177-201.

[14] M. Vohralík. A posteriori error estimation in the conforming finite element method based on its local conservativity and using local minimization. C. R. Math. Acad. Sci. Paris, 346 (2008), 687-690. 